\title{
PREPRINT
}

\section{Examining the Impact of Working Memory Training and Transcranial Direct-current Stimulation on Monitoring for Color Targets in Dynamic Visual Displays}

\author{
Alex Muhl-Richardson, Hayward J. Godwin, Matthew Garner, Julie A. Hadwin, \\ Simon P. Liversedge and Nick Donnelly \\ University of Southampton, UK
}

\author{
ARTICLE HISTORY \\ Compiled May 23, 2019
}

\begin{abstract}
Previous research has demonstrated that when monitoring dynamically changing visual displays for color targets, contingencies between targets and distractors facilitate predictive target detection, and elevated intolerance of uncertainty (IU) and lower verbal working memory capacity (WMC) are associated with a higher false alarm rate (see Muhl-Richardson et al., 2018). The present study examined whether search in this type of task improved following placebo-controlled adaptive dual n-back working memory (WM) training or transcranial direct-current stimulation (tDCS) of the dorsolateral prefrontal cortex. It also attempted to replicate previous findings but at a high level of target prevalence. The results demonstrate a new benefit to predictive target detection, which was associated with faster response times, and identify elevated IU and verbal WMC were as significant predictors of a higher hit rate. We provide an account of these effects in terms of response criterion and perceptual sensitivity. WM training and tDCS were found not to benefit performance on our dynamic search task (and a range other cognitive tasks), adding to a mixed literature on the efficacy of these techniques. The results nonetheless extend our understanding of dynamic search tasks and have implications for the selection of personnel in a variety of real-world scenarios.
\end{abstract}

\section{KEYWORDS}

Visual search; eye movements; dynamic search; working memory training; transcranial direct-current stimulation

\section{Introduction}

Spatial and verbal working memory capacity (WMC) have been associated with performance in challenging visual search and target detection tasks (Anderson, Mannan, Rees, Sumner, Kennard, 2008; Schwark, Sandry, Dolgov, 2013; Woodman Luck, 2004). In individuals who report elevated anxious affect, WMC can be consumed when

Author note: Alex Muhl-Richardson is now at the Department of Psychology, University of Cambridge; Simon P. Liversedge is now at the Department of Psychology, University of Central Lancashire; Nick Donnelly is now at the Department of Psychology, Liverpool Hope University.

This research was conducted as part of the Defence Science and Technology Laboratory National PhD scheme. The authors would like to thank Nazanin Derakshan and colleagues in the Department of Psychological Sciences at Birkbeck, University of London for their assistance and provision of working memory training.

Correspondence regarding this article should be addressed to Alex Muhl-Richardson, Department of Psychology, University of Cambridge. E-mail: am2662@cam.ac.uk 
attentional resources are directed at task-irrelevant internal (e.g. worrisome thoughts) and external (e.g. potential negative feedback from others) information (see reviews by Derakshan Eysenck, 2009; Eysenck Derakshan, 2011; Eysenck, Derakshan, Santos, Calvo, 2007; Richards, Hadwin, Benson, Wenger, Donnelly, 2011). In individuals with low WMC, this can leave little capacity remaining for allocation to other tasks. Training or otherwise improving WMC therefore represents a potential means for alleviating some the cognitive and attentional costs associated with anxious affect.

In a previous study (Muhl-Richardson et al., 2018), we found evidence of predictive target detection and active monitoring of changing stimuli in a dynamic color search task. We also reported that target detection in this task was influenced by individual differences in verbal WMC and intolerance of uncertainty (IU), a component of trait anxiety that relates specifically to uncertain future events (Birrell, Meares, Wilkinson, Freeston, 2011) and which has been shown to influence attention even when controlling for anxiety, depression, and distress (Fergus, Bardeen, Wu, 2013; Fergus Carleton, 2016). The search task used in these experiments involved displays of fixed-location colored squares that changed color dynamically over time and differs from some other dynamic search tasks which involve dynamically moving stimuli (e.g. Kunar Watson, 2011, 2014; Scarince Hout, 2018). The task was designed to simulate the monitoring for target onsets in complex visualization displays used in the real world (e.g. marine radar, volumetric geological imaging, meteorological mapping, etc.).

Our previous work found evidence that symptoms of IU were positively associated with false alarm rate such that distractor colors were mistakenly classified as targets. The relationship of IU and false alarms was moderated by verbal WMC, such that increased WMC reduced the effect of IU on the false alarm rate. The interaction between IU and WM was also found in eye movements recorded during the task: individuals high in IU and verbal WMC made more fixations, suggestive of more extensive searching and monitoring. This finding is consistent with the compensatory role of WM outlined by theoretical frameworks of anxiety and cognition (Derakshan Eysenck, 2009; Eysenck Derakshan, 2011; Eysenck et al., 2007), which suggest that the impact of anxiety on attentional control may be most evident in individuals with limited WM resources. The interaction between IU and WMC also fits with a broader pattern of results which highlight the importance of attentional control and WM in understanding the cognitive effects of anxiety and suggest a protective role for these factors that moderates the impact of elevated anxiety on cognitive processing (Booth, Mackintosh, Sharma, 2017; Hadwin, Visu-Petra, Muris, Derakshan, MacLeod, 2016; Matthew Owens, Stevenson, Hadwin, Norgate, 2014).

The relationship between these factors reveals a potential target for training or other methods to improve target detection in dynamic search tasks, not by attempting to change stable anxiety-related traits, but with techniques that might enhance WMC. If individual differences in WMC can be assessed and targeted in a way that not only improves WMC but also mitigates the negative effects of anxiety-related traits, then there is a strong possibility of implementing such an approach to improve performance in a range of real-world visual search and monitoring tasks. Having previously found evidence that IU and WMC predicted performance in our dynamic search task, in the present study we focus on two possible methods for improving target detection in this task, selected due to their potential benefits to WM: (1) a program of WM training; and (2) a session of transcranial direct-current stimulation (tDCS). 


\subsection{Working Memory Training}

WM training typically involves a computerized adaptive complex span or n-back type task that loads heavily upon WM (see reviews by Au et al., 2014; Au, Buschkuehl, Duncan, Jaeggi, 2015; Melby-Lervg Hulme, 2015). Participants complete these tasks regularly and over multiple days, while the difficulty of training is adapted to individual performance in terms of the number of items or steps to be remembered. Training is typically assessed by performance improvements within the training sessions, as well as benefits to untrained tasks.

The near transfer of benefits to untrained tasks that are similar to the training task is commonly reported (e.g. Brehmer, Westerberg, Bckman, 2012; Klingberg, Forssberg, Westerberg, 2002; Westerberg Klingberg, 2007), but the evidence supporting more distant transfer to tasks that capture the broader generalization of training effects is more mixed. While many studies have failed to obtain significant training benefits of this type (review by Shipstead, Hicks, Engle, 2012), some key studies have shown improved fluid intelligence (Jaeggi, Buschkuehl, Jonides, Perrig, 2008; Jaeggi, Buschkuehl, Jonides, Shah, 2012) and attentional control (Max Owens, Koster, Derakshan, 2013) following WM training. For the present study a dual n-back WM training task was selected (Max Owens et al., 2013; Sari, Koster, Pourtois, Derakshan, 2015) that was developed based upon the task used by Jaeggi et al. (2008). This task includes simultaneous auditory and visual components, has a training period of approximately two weeks and has been used to demonstrate significant improvements to WMC in a training group of 11 participants (Max Owens et al., 2013).

\subsection{Transcranial Direct-current Stimulation}

The second method examined in this study was tDCS, a non-invasive brain stimulation technique which modulates cortical excitability via the delivery of a very weak electrical current (typically between 1 and $2 \mathrm{~mA}$ ) through electrodes placed on the scalp (Nitsche et al., 2008). Early research showed that neuronal depolarization at the anodal stimulation site resulted in a localized increased likelihood of neuronal firing and a converse effect at the cathodal stimulation site (Nitsche Paulus, 2000) and, while much of this early research involved stimulation of the motor cortex (e.g. Nitsche Paulus, 2000, 2001; Rosenkranz, Nitsche, Tergau, Paulus, 2000), this technique has since been applied to other brain regions, including prefrontal areas. The dorsolateral prefrontal cortex (DLPFC) has important roles in high level cognition, including WM and the executive control of attention (Lara Wallis, 2015; Rossi, Pessoa, Desimone, Ungerleider, 2009), and has often been targeted in tDCS studies with a view to improving these functions (Tremblay et al., 2014). Stimulation of the DLPFC has been associated with a wide range of outcomes, including improvements in associative learning (Kincses, Antal, Nitsche, Brtfai, Paulus, 2004), recognition memory (Pergolizzi Chua, 2017; Smirni, Turriziani, Mangano, Cipolotti, Oliveri, 2015; Zwissler et al., 2014), verbal fluency (Iyer et al., 2005), anxiety-related attentional bias (Clarke, Browning, Hammond, Notebaert, MacLeod, 2014), risk taking behavior (Fecteau et al., 2007), processing emotional stimuli (Boggio et al., 2007), WM (Berryhill Jones, 2012; Fregni et al., 2005; Keeser et al., 2011; Mulquiney, Hoy, Daskalakis, Fitzgerald, 2011; Teo, Hoy, Daskalakis, Fitzgerald, 2011) and the executive control attentional network (Miler, Meron, Baldwin, Garner, 2017). We specifically examine the efficacy of a bilateral stimulation montage with the anode over the left DLPFC (and cathode over the right DLPFC), relative to a sham control, as this electrode configuration has 
been shown to afford benefits to WM (Oliveira et al., 2013; Saggini et al., 2016).

\subsection{The Present Study}

While the focus of the present study is to examine the influence of WM training and tDCS on performance in the dynamic visual search task, this task involves a complex set of cognitive demands and, in terms of the WM training literature, represents a distant transfer task. To maximize sensitivity to the effects of WM training and tDCS, and to ensure their efficacy in terms of near transfer, we also include a battery of WM and attentional tasks. In the event that the benefits of training/stimulation to do not transfer to the more complex dynamic visual search task, we would still reasonably expect to observe effects in these simpler tasks.

An additional factor that might influence the nature of the effects observed in the dynamic visual search task is target prevalence. Target prevalence is known to influence the speed and accuracy of responses in visual search experiments and can also influence when participants stop searching (Wolfe et al., 2007; Wolfe, Horowitz, Kenner, 2005). A source of this effect is via a criterion shift that influences the identification of targets (Godwin, Menneer, Riggs, Cave, Donnelly, 2014; Wolfe Van Wert, 2010). In other words, when targets are rare, individuals require more perceptual evidence to make a target-present response, resulting in misses and slowed responses, and the converse is true when targets are common. In visual search studies target prevalence is typically defined as the percentage of target present trials. However, this is not the optimal way to consider target prevalence in tasks with extended trial durations, where a target may be present in a trial, but only for a small proportion of the duration of that trial.

In the present study, we employ a novel conception of target prevalence, in terms of the proportion of the total trial time for which targets are present. This provides a better starting point for understanding the role of prevalence within this class of task and reveals that, even when targets are present on $66 \%$ of trials, target prevalence can still be low. In order to avoid the effects related to low target prevalence described above, we employ a significantly higher overall level of target prevalence than in our previous study with this task.

We believe that target prevalence may have an effect that is unique to search tasks with dynamically changing stimuli that cannot be observed in static tasks. Specifically, when target prevalence is high, potentially target-predictive stimuli have a high probability of being valid cues to a target onset and predictive target detection is therefore more likely to be beneficial compared to simply detecting target onsets. This novel type of prevalence effect would explain previous findings under low target prevalence where predictive detection was not associated with a behavioral benefit and could influence the nature of the three-way association between target detection, WMC and IU.

In summary, the present study has three main goals: (1) to examine how benefits to WMC following WM training or tDCS improve target detection by moderating the impact of IU; (2) to confirm the interaction between IU and verbal WMC with respect to target detection; and (3) to examine how predictive target detection is influenced by a higher overall level of target prevalence. These goals will be addressed primarily using a novel dynamic visual search task during which eye movement data will be recorded to complement behavioral outcomes. Data from a battery of memory and attentional tasks performed prior to and after training/stimulation will also be reported to confirm near transfer effects. It is predicted that previous eye movement evidence of predictive 
target detection and the association between IU and false alarm rate (moderated by verbal WMC) will be replicated. It is further predicted that active WM training and tDCS (relative to controls) will provide a behavioral benefit to target detection in terms of either hit rate or false alarm rate and that any such benefit will be greater for those with greater symptoms of IU due to the moderating effect of WMC. The high level of target prevalence in the present task is expected to improve behavioral performance relative to previous results (i.e. increased hit rate, and reduced response time and false alarm rate).

\section{General Method}

\subsection{Design}

In the case of both the WM training and the delivery of tDCS, the primary independent variable was whether the WM training/tDCS was active or a placebo/sham control and this was manipulated between participants. Participants were tested on the search and monitoring task at two times, baseline and post WM training/tDCS. The primary dependent variables for the search and monitoring task included behavioral measures of response time (RT), hit rate and false alarm rate, and eye movement measures of the proportion of first fixations on targets and potentially target-predictive distractors that were target-similar colors, the number of fixations, fixation duration and scanpath length. In order to explore the near and distant effects of both WM training and tDCS on WMC and attentional control, additional measures included a visual digit span, a visuo-spatial change detection task, an antisaccade task and the Attention Network Test (ANT). IU was also recorded to explore the relationship between task performance and individual differences in this trait. All experiments were approved by the Psychology Ethics Committee at the University of Southampton and by the Ministry of Defence Research Ethics Committee.

\subsection{Apparatus and Materials}

For all computerized tasks completed in the laboratory, stimuli were displayed on a 21 CRT monitor operating at a resolution of $1,024 \times 768$ and a refresh rate of $120 \mathrm{~Hz}$. The visual search and monitoring task and antisaccade tasks were controlled using a computer connected to an SR Research EyeLink 1000 eye-tracker operating at a sampling rate of 1,000 Hz. The search and monitoring task was administered using the same procedure as in (Muhl-Richardson et al., 2018). A nine-point calibration procedure was used and calibration was accepted only when none of the points had an error of more than 0.5 of visual angle. For the antisaccade task, a three-point calibration procedure was used and calibration was accepted only when none of the points had an error of more than 1.0 of visual angle. For both of these tasks, participants were seated $70 \mathrm{~cm}$ from the display, the display was viewed binocularly, only the right eye was tracked, and a chin rest was used to keep participants heads stable. These two tasks were programmed using Experiment Builder from SR Research with additional code written in Python. 


\subsection{Search and Monitoring Task}

Stimulus displays were generated on a trial-by-trial basis and all angles in this section are visual angles calculated from the centre of the display (values vary marginally for more eccentric locations). Individual colored squares (items) were $0.57 \times 0.57$, and displayed in irregular $12 \times 9$ arrays. Items never abutted, but were randomly jittered within the constraints of an invisible grid, such that each stimulus appeared within an area of 2.15 by 2.15 , which was used to code fixations. The maximum size of the whole array of color stimuli was 24.78 x 18.36 and 108 items were always present.

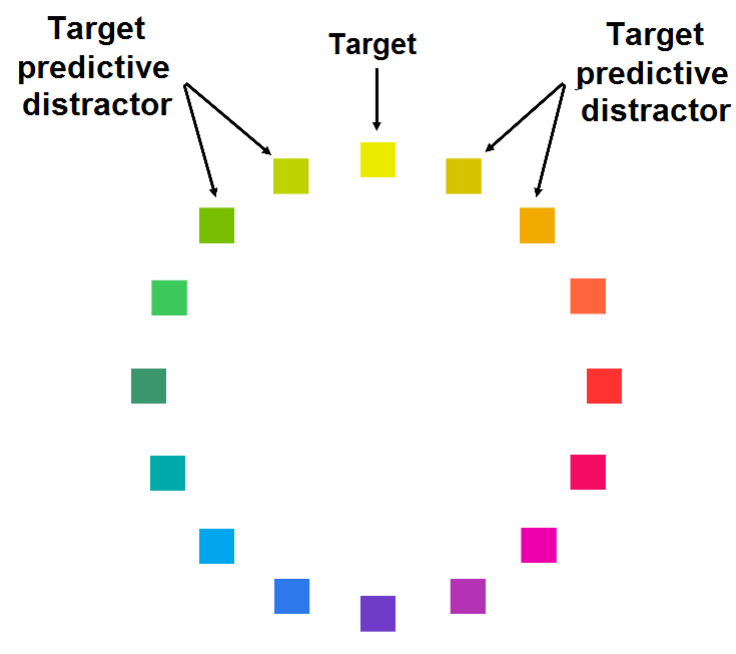

Figure 1. Full color scale showing example target predictive distractors when the target was the indicated color.

Stimuli were colored using a 16-item color scale (see Menneer et al., 2007). For each participant, one color was designated as the target (T) and 15 colors as distractors (D). Of the 108 colored squares, 96 squares were always at least 3 steps away from being a target and these were considered background distractors. There were 12 squares that could display colors within 2 color steps of the target. The locations of these 12 squares were varied across trials and, unknown to participants, these were never located at the edge of the array or immediately adjacent to another of these 12 squares. The target color was varied and counterbalanced between participants, such that each color was the target for at least one participant (Figure 1 shows the full color scale).

The search and monitoring task was always administered in a single 72-trial block with a self-paced break at the halfway point and, within this, 36 trials had up to five targets present and 36 trials were target absent. The total number of targets presented over the course of each target present trial was dependent upon participant performance and the restriction that no more than one target could be present at any one time. Failing to detect an earlier target could delay (or in rare cases exclude) the onset of later targets. Participants were shown a static sample display (see Figure 2) and were given a practice block of three trials before beginning the task. Trials began with a $1 \mathrm{~s}$ reminder of their target, followed by a $1 \mathrm{~s}$ fixation point. The stimulus display was present for $40 \mathrm{~s}$ (see Figure 3) and audio feedback was given for every response (to indicate whether it was correctly made to a target or not). Participants 
were told to search for a target square defined by a particular color, which was shown before every trial, and to respond as quickly as possible by clicking the mouse cursor on the target. Participants were told that trials might contain more than one target but only one target would be visible at any given time

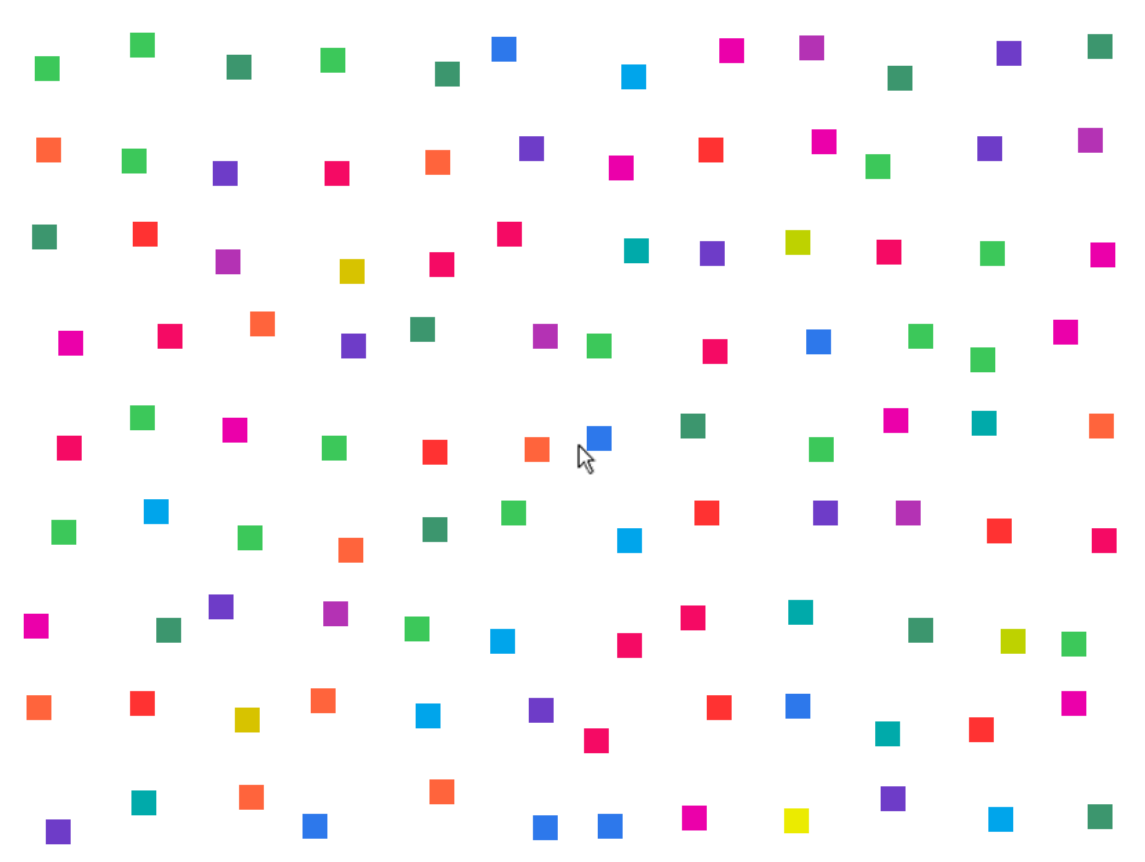

Figure 2. Sample stimulus display.

Trials began with all items as background distractors. Stimulus arrays were updated with a variable refresh rate, which resulted in displays that refreshed at a mean rate of once every $105 \mathrm{~ms}(\mathrm{SD}=96)$ and the mean rate of color change for each item was a change every $339 \mathrm{~ms}(\mathrm{SD}=352)$. Background distractors in both conditions could change between the 11 colors that were at least three steps from the target color. When changing color, items could change to be closer to, or further from, the target color. For background distractors, these two possibilities were equally likely, other than when three steps from the target color. At certain time points during trials, the 12 squares that could display colors within two steps of the target were allowed to change across the full range of 16 colors (or 15 colors if they were not to become a target). As there were up to five targets per trial, at least seven of these 12 stimuli reached a color one step from the target, but never achieved a target state. The effect of these conditions was to allow stimuli to systematically approach the target color, allowing the color of distractors to be used as a predictive cue to the onset of targets.

Each of these 12 items was given a number of key properties, all independently and pseudo-randomly generated within Python: (1) a time at which they were allowed to move within two colors of the target; (2) a time after which they will become a target or a distractor color one step from the target; and (3) a duration for which they remain present as target or a distractor one step from the target before reverting to 


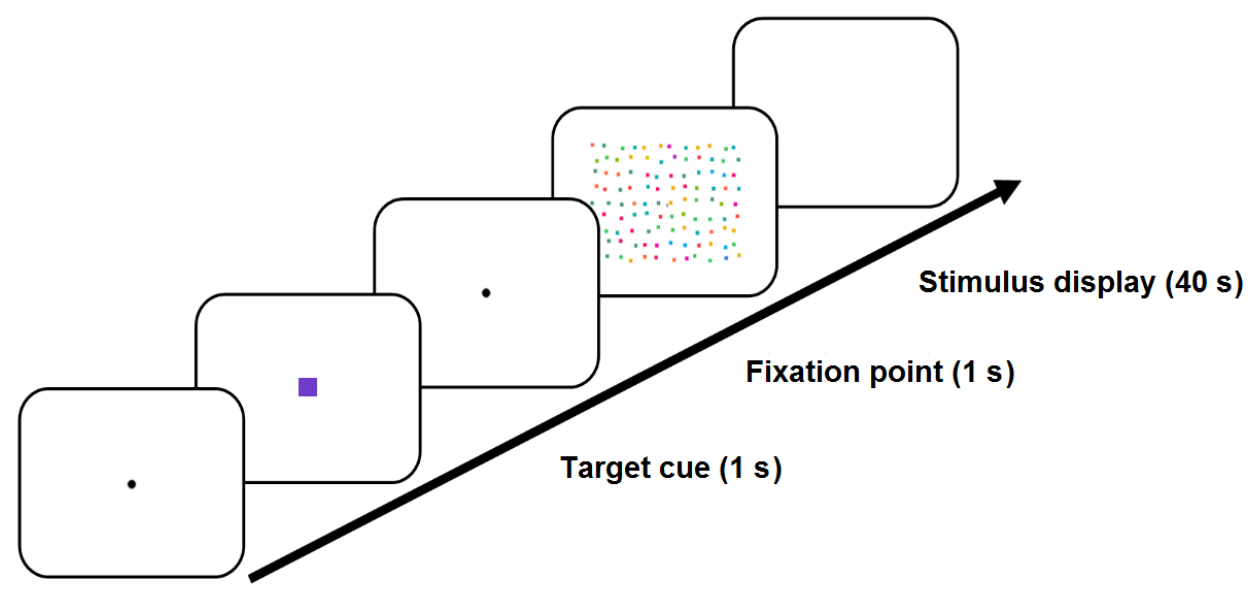

Figure 3. Trial sequence.

background distractor (if no response was made). These values corresponded to the first target onset after an average of $9,362 \mathrm{~ms}(\mathrm{SD}=3,929)$. On average and where applicable, the second target onset occurred after 14,860 ms $(\mathrm{SD}=4,490)$, the third target onset occurred after $20,159 \mathrm{~ms}(\mathrm{SD}=5,441)$, the fourth target onset occurred after $24,453 \mathrm{~ms}(\mathrm{SD}=5,266)$ and the fifth target onset occurred after 29,076 $\mathrm{ms}(\mathrm{SD}=$ $5,193)$. This also resulted in an average duration of targets, if no response was made, of $1,347 \mathrm{~ms}(\mathrm{SD}=739)$. If no response was made to a target, the item timed-out and was reset to a background distractor. Responding target-present (irrespective of whether it was a hit or a false alarm) also caused it to reset to a background distractor. While up to five targets might be presented over the course of a trial, only one target could be present at any given time. The parameters that controlled stimulus behavior were selected based on previous work with the same task (Muhl-Richardson et al., 2018).

\subsection{Working Memory Capacity and Attentional Control Tasks}

\subsubsection{Visual digit span}

The digit span task used was developed by Borchert (2016) and follows the procedure detailed in Woods et al. (2011). In each trial of this task a sequence of digits (0 to 9 inclusive) was presented one-at-a-time in the center of the display and, when a sequence had finished, participants were required to enter the digits in a text box using the keyboard (see Figure 4). The task was split into two blocks, in the first block participants were required to enter digits in the same order in which they were presented, in the second block digits needed to be entered in reverse order to presentation. If the sequence of digits was entered correctly, then the sequence length was increased by one digit. If the sequence of digits was entered incorrectly twice consecutively, the task continued, but the sequence length was reduced by one digit (not below the starting level). In the forward block, the sequence length began at three digits and in the backward block at two digits. Participants were given a single practice trial at the initial sequence length before beginning each block and each block contained at least 14 trials (contingent upon performance). Performance was reflected in the maximum sequence length recalled correctly before making two consecutive errors. 


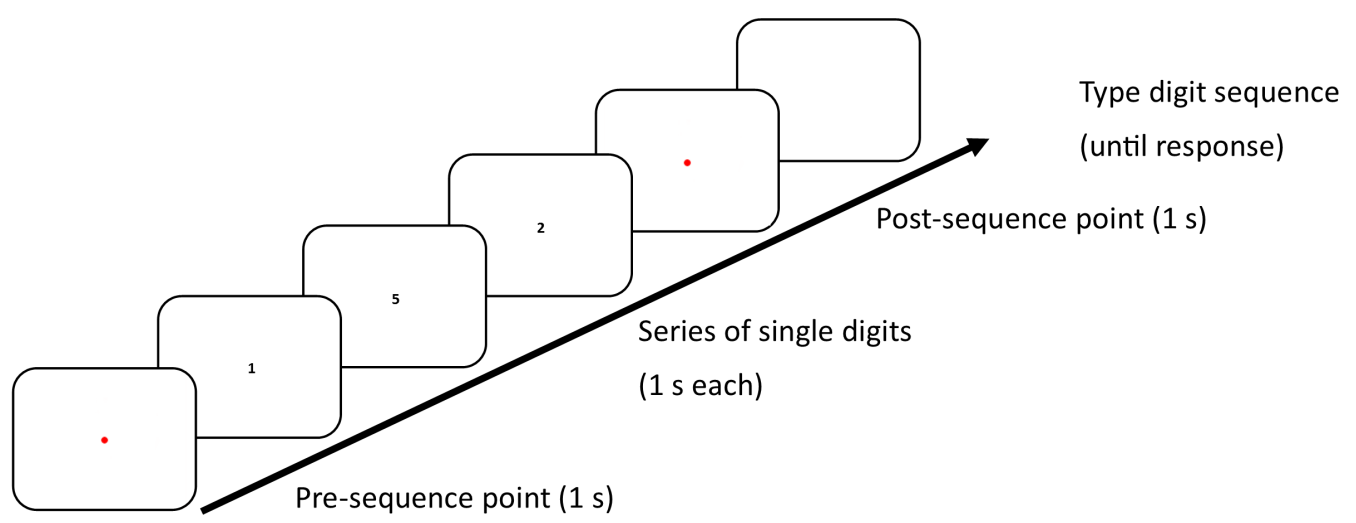

Figure 4. Visual digit span trial sequence.

\subsubsection{Change detection task}

This task follows the procedure used by Owens et al. (2012; 2013). Trials involved two stimulus arrays, a memory array and a test array, which consisted of red and blue rectangles (see Figure 5). Participants were required to remember the orientation of target items (red stimuli), from either the left or right side of the memory array and respond, with one of two button presses, to indicate whether or not the orientation of one of these stimuli had changed in the test array. $50 \%$ of trials involved a change and stimuli were viewed at a distance of $70 \mathrm{~cm}$. Each array consisted of two regions (5.99 x 9.04), each containing two or four rectangles. Regions were centered 4.22 left and right of a central fixation cross. Each rectangle was randomly assigned one of four orientations (vertical, horizontal, left 45, right 45) and could be either red (target) or blue (distractor). Each trial began with a central fixation cross and a white arrow cue, pointing left or right, visible for $700 \mathrm{~ms}$. Participants were instructed to maintain fixation on the cross during each trial, but to attend to the side indicated by the arrow. After presentation of the cross and arrow, the left and right regions showed 2 red rectangles, 4 red rectangles or 2 red rectangles and 2 blue rectangles for $100 \mathrm{~ms}$ (memory array). Only the cross was then displayed for $900 \mathrm{~ms}$ (retention period) and then the rectangles were redisplayed for 2,000 ms (test array). The inter-trial interval was randomly set at either $1,500 \mathrm{~ms}$ or 2,000 ms. Array size, arrow direction, change and no-change trials were randomized and had equal prevalence across the experiment. Participants completed a practice session of 24 trials ( 8 per condition) before the main task. The task was split into 4 blocks of 48 trials (64 trials per condition), totaling 192 trials across the experiment. A short break was given after each block. Performance was reflected in the proportion of correct responses (hit rate).

\subsubsection{Attention Network Test}

The Attention Network Test (ANT) combines a flanker task and a cued reaction time task to provide measures of attentional orienting, altering and executive control (Fan, McCandliss, Sommer, Raz, Posner, 2002; Fan, McCandliss, Fossella, Flombaum, Posner, 2005). In the present case, this task consisted of 8 randomized practice trials followed by 64 randomized experimental trials. On each trial a central fixation cross 


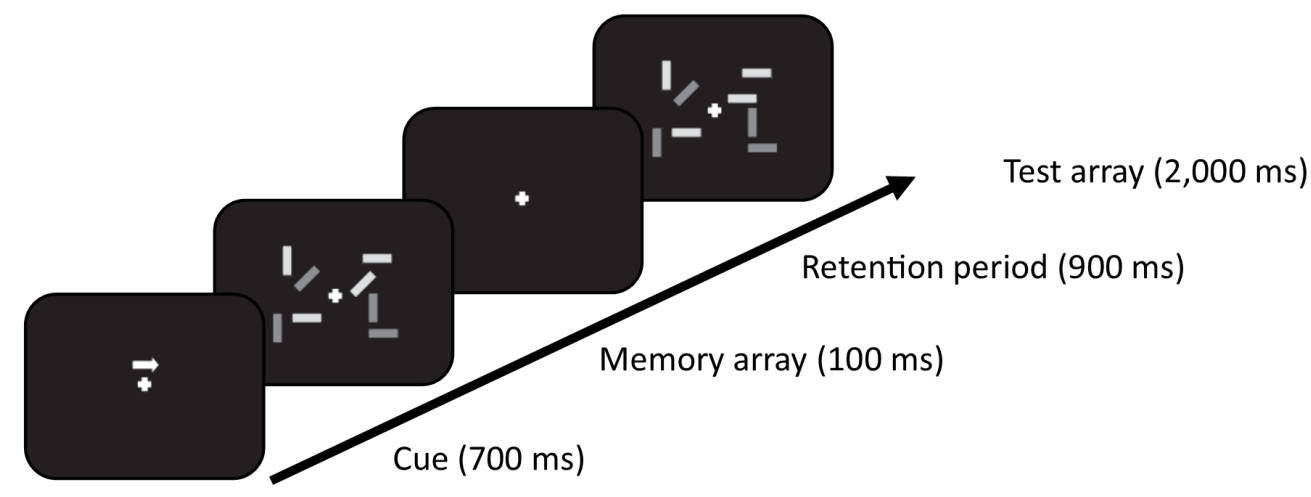

Figure 5. Change detection task trial sequence (figure adapted from Owens et al. [2012]); red rectangles are shown as light grey and blue as dark grey; a response was made during the test array.

was presented for 4001,600 ms, followed by a cue for $100 \mathrm{~ms}$ (except on no-cue trials). Subsequently, $400 \mathrm{~ms}$ after cue offset (or $500 \mathrm{~ms}$ in no-cue trials), target and flanker arrows were displayed until the participant made a manual button press response. On double cue trials the cue was displayed both above and below the fixation cross and alerted participants to the onset of the target (but not spatial location). On center cue trials the cue was displayed in the location of the fixation cross and alerted participants to the onset of the target. In contrast on spatial cue trials the cue was displayed above or below the fixation cross and signaled both the onset and spatial location of the target (always displayed in the location of the cue), thus orienting attention towards the location of the pending target. Participants were instructed to classify as quickly and accurately as possible whether the central (target) arrow pointed left or right. The target arrow was flanked by two pairs of distracter arrows. Flanker arrows either pointed in the same direction (congruent condition) or opposite direction (incongruent condition) as the target arrow. Flanker congruence, target direction and target location were counterbalanced across spatial, double, center and no-cue trials and RTs were collected via keyboard button press. The task sequence and all cue and target types are shown in Figure 6.

\subsubsection{Antisaccade task}

The antisaccade task used here was adapted from that used by Hepsomali, Hadwin, Liversedge and Garner (2017). It consisted of a short practice block of 10 trials and then two blocks of 100 trials. Each trial began with the presentation of central letter cue, $\mathrm{O}$ or $\mathrm{X}$, to indicate whether a pro- or antisaccade should be made respectively (see Figure 7). This was then replaced with a fixation cross which remained visible for either 1,000 ms or 3,000 ms, before a grey ellipse $(4.59 \times 3.66)$ appeared on either the extreme left or right hand side of the display. The side of the display where the ellipse was presented, the delay and the type of saccade required were counterbalanced and the order of trials was randomized. On prosaccade trials participants were instructed to look at the ellipse and on antisaccade trials participants were instructed to look at the opposite side of the screen. The ellipses were displayed for 1,500 ms. Performance 


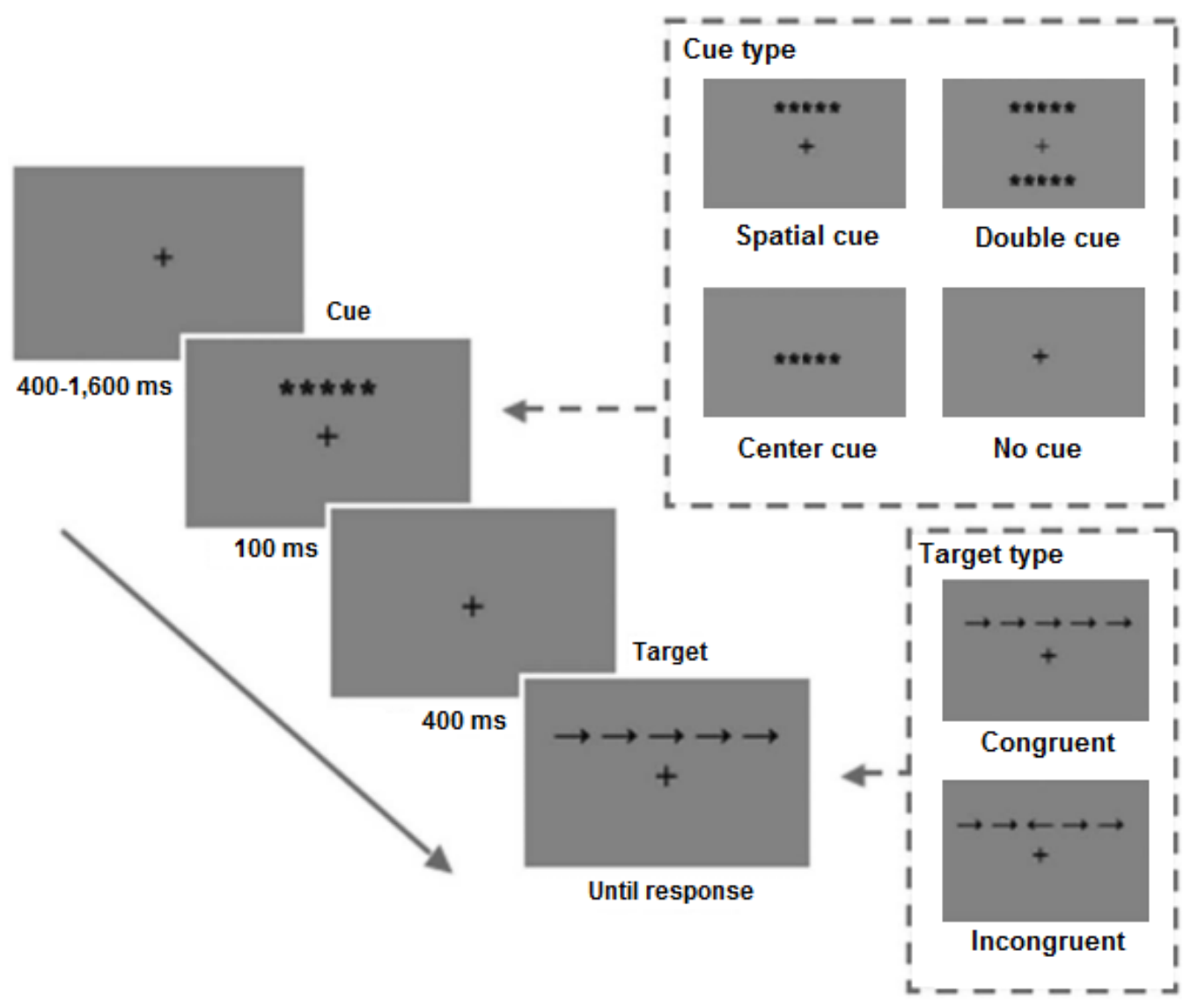

Figure 6. Attention Network Test sequence with all cue and target types (figure adapted from Garner et al. [2012]).

was measured in terms of the accuracy (whether the correct type of saccade was made) and latency of the first saccade made after stimulus onset.

\subsection{Anxiety and Mood Measures}

\subsubsection{Intolerance of uncertainty}

The Intolerance of Uncertainty Short Form (IUS-12; Carleton et al., 2007) is a 12item self-report measure that assesses reactions to uncertainty, ambiguous situations and future events. It consists of two factors, the first, prospective IU, relates to worry regarding future events and is assessed by 7 items, for example, I cant stand being taken by surprise. The second, inhibitory IU, relates to the extent to which uncertainty might inhibit action or experience and is assessed by 5 items assessing, for example, when its time to act, uncertainty paralyses me. Each response is made on a five-point scale which ranges from 1 not at all characteristic of me to 5 entirely characteristic of me and this results in a total score between 12 and 60 . In the current study the reliability of the scale was good $(i=.81)$. 


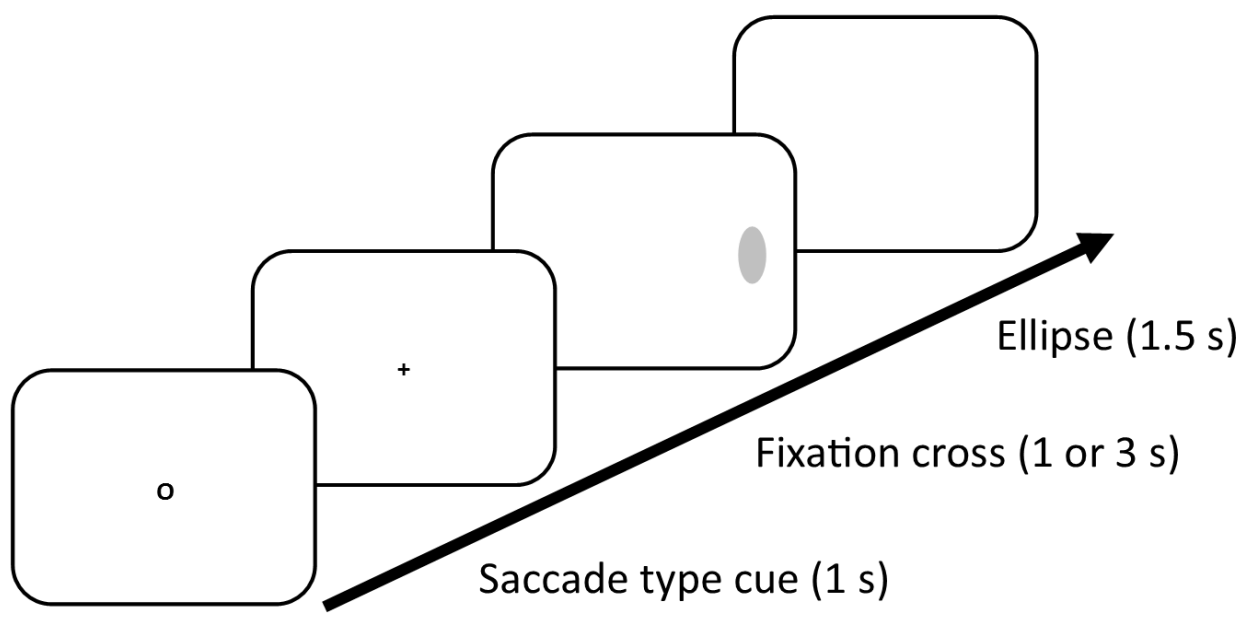

Figure 7. Anti-saccade task trial sequence (pro-saccade trial illustrated).

\subsubsection{Visual analogue scales}

We used visual analogue scales (VAS) to measure participants mood at three time points during both visits: (1) before completing the search and monitoring task; (2) after completing the search and monitoring task; and (3) at the end of the session. The VAS asked participants to rate the extent to which they felt anxious, alert, happy, able to concentrate, relaxed, worried and tired. For the second and third VAS of each session, a further item was included that asked participants how hard they tried. Participants responded to each VAS item by making a mark on a dotted line, allowing for continuous responses, however, for guidance, each line was labelled (from left to right) not at all, a little, moderately, quite a bit and extremely. Each item was scored between 0 and 160 using the distance of the response along the line.

\subsection{Procedure}

Participants were tested for normal visual acuity and color vision using the Freiburg Visual Acuity Test (Bach, 1996) and the City University Color Vision Test (3rd edition; 1998). To maximize comparability between the WM training and tDCS, the same safety screening was applied to all participants before they were allowed to enter the study, this involved a brief medical history and a neuropsychiatric interview based on the M.I.N.I. International Neuropsychiatric Interview (Sheehan et al., 2006).

Participants made two visits to the laboratory. The same procedure was followed on each visit with the exceptions noted in the sections below. Participants completed the IUS-12 before completing the search task on the first visit ${ }^{1}$. After the search and monitoring task, participants completed the antisaccade task, the ANT, the digit span task and the change detection task in that order. Self-timed breaks were given

\footnotetext{
${ }^{1} \mathrm{IU}$ is considered a stable trait and we expected that IUS-12 scores would remain stable between visits. Participants were asked to complete the IUS-12 again during their second visit, but as scores did not differ significantly between the two time points, only the data from the first visit was analysed.
} 
to participants between blocks in the antisaccade task and before starting each new task.

\subsection{Training Working Memory}

\subsubsection{Participants}

20 students (14 female) from the University of Southampton participated in the WM training arm of the study for partial fulfillment of a course requirement or for compensation of 78 . This sample size was selected to be marginally greater than the average sample size reported in a recent meta-analysis (Au et al., 2014, 2015) and similar to that in study which used the same training task and observed significant benefits to WMC following training (Max Owens et al., 2013). Their mean age was 20.95 years ( $\mathrm{SD}=2.37$; Range 19 to 26 years). All participants had normal visual acuity (at least 1.0 decimal VA at $70 \mathrm{~cm}$ ), tested using the Freiburg Visual Acuity Test (Bach, 1996) and normal color vision, tested using the City University Color Vision Test 3rd Edition (Fletcher, 1998).

\subsubsection{Training Task}

A dual n-back WM training task was used (Max Owens et al., 2013; Sari et al., 2015) based on a task originally developed by Jaeggi et al. (2008). In the task, participants were shown a $3 \times 3$ grid, with a fixation cross in the center. In each trial, a green square appeared in one of the eight outermost locations in this grid and, paired with each presentation of a square (within $500 \mathrm{~ms}$ ) one of eight consonants (c, h, k, l, q, $\mathrm{r}, \mathrm{s}$, and $\mathrm{t}$ ) was spoken (see Figure 8). There was a delay of 2,500 ms between trials. Participants were told to remember both the location of the green square within the grid and the letter that they had heard and to respond if either of these stimuli matched a letter or location presented $\mathrm{n}$ trials back in the sequence. Participants responded using a keyboard and pressed the A key for visual matches and the L key for auditory matches (sometimes the correct response was to press both of these at the same time). No response was required if there was no match and participants were told to respond as quickly and accurately as possible. Participants completed this dual n-back task online in their own time using a computer of their choosing. The task was administered and managed via a bespoke website that allowed the experimenter to monitor training progress. This website was developed and hosted by the Department of Psychology at Birkbeck, University of London.

In the adaptive training condition, participants in the training group completed 20 blocks of $20+\mathrm{n}$ trials, where $\mathrm{n}$ was determined by the level of n-back (e.g., 2back, $20+2=22$ trials). Within the adaptive condition, the n-back level (which determined task difficulty) was varied based on the percentage accuracy across visual and auditory responses after each block. Each training session always began with a block at the 1-back level and after this block, and for every subsequent block in that session, the level of $\mathrm{n}$ was increased by one if accuracy was at or above $95 \%$ for both modalities, decreased by one if accuracy was below $75 \%$ in either modality and remained unchanged if accuracy was between $75 \%$ and $95 \%$. The 4-back level was set as the highest n-back level in the study. Participants were reminded of the n-back level before each block and this reminder remained visible throughout. In the non-adaptive control condition, the n-back level was fixed at 1-back. Participants completed 20 blocks of 20 trials per session. This was to ensure that the control group remained active 
and performed a similar task to the adaptive training group, but with minimal reliance upon working memory processes. To ensure an equal number of participants in each training condition while maintaining randomization, group assignment was conducted using a randomly shuffled list. Participants were blind to their group assignment.

\subsubsection{Training Procedure}

Immediately prior to leaving the laboratory at the end of the first visit, all participants completed a practice block of 20 trials on the task at the 1-back level. Later that day, and in their own time, participants completed the first full session of training. In total, all participants completed 15 training sessions, each consisting of 400 trials divided equally across 20 blocks and lasting approximately 30 minutes (participants in the adaptive training condition completed some additional trials dependent upon n-back level). Each block lasted approximately one minute, with a break of 15 seconds was given between blocks, and training sessions could not be paused once started. Targets were varied randomly such that each block had an equal number of visual and audio matches (4 per block) and two trials with a double (auditory and visual) match. Target positions within blocks were also varied randomly such that the value of $n$ was the same for visual and auditory stimuli.

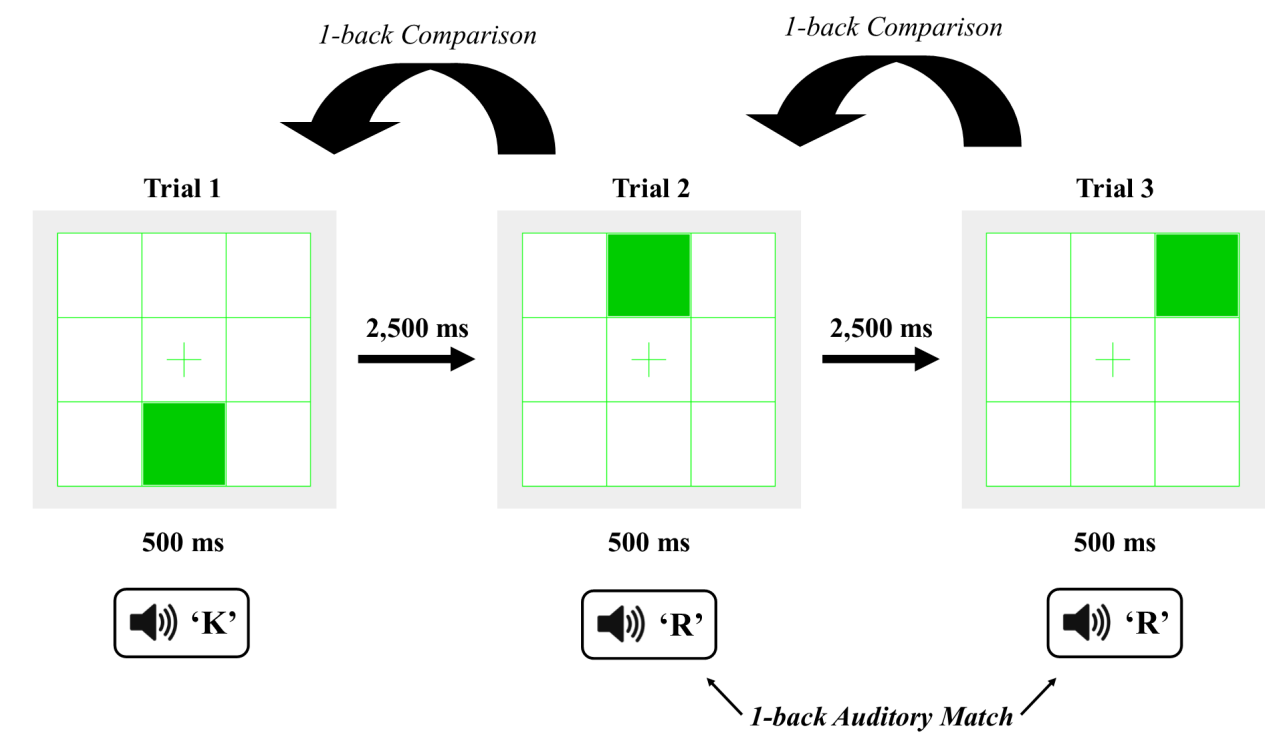

Figure 8. A sequence of three trials in the training task at the 1-back level. The correct response would be to press $\mathrm{L}$ on the third trial for the auditory match with the second trial.

Participants completed the training over a period of 15 to 17 days, such that no more than one training session was completed per day but participants could take up to two break days. Participants second visit to the laboratory for their post-training assessment was always on the same day as or the day following the final training session. Training performance was monitored by the experimenter and participants were shown a summary of their auditory and visual scores over the session at the end of each block. Figure 5 shows the overall set of study procedures. 


\subsection{Transcranial Direct-current Stimulation}

\subsubsection{Participants}

26 students (18 female) from the University of Southampton participated in the tDCS arm of the study for partial fulfilment of a course requirement or for compensation of 39. This sample size was selected to be greater than or similar to studies reporting significant benefits to WM following anodal stimulation of the left DLPFC (Boggio et al., 2006; Fregni et al., 2005; Ohn et al., 2008), including studies with a bilateral stimulation montage like the one used in the present study (Oliveira et al., 2013; Saggini et al., 2016). It is also between the average sample sizes reported in two recent meta-analyses examining the effects of prefrontal tDCS on a variety of cognitive tasks (Brunoni Vanderhasselt, 2014; Dedoncker, Brunoni, Baeken, Vanderhasselt, 2016). Their mean age was 21.27 years ( $\mathrm{SD}=2.55$; Range 18 to 27 years). All participants had normal visual acuity (at least 1.0 decimal VA at $70 \mathrm{~cm}$ ), tested using the Freiburg Visual Acuity Test (Bach, 1996) and normal color vision, tested using the City University Colour Vision Test 3rd Edition (Fletcher, 1998).

\subsubsection{Stimulation Parameters}

Stimulation was administered bilaterally, with the anode over the left DLPFC and the cathode over the right DLFPC (F3 and F4 respectively according to the 10-20 EEG system). Compared to unilateral stimulation, bilateral stimulation reduces the uncertainty around differences in the interhemispheric balance of activity between participants (Fecteau et al., 2007; Tremblay et al., 2014). Further, bilateral stimulation with the anode over the left DLPFC (and cathode over the right DLPFC) has been shown to benefit WM (Oliveira et al., 2013; Saggini et al., 2016). In the active condition, participants received stimulation with a current of $2 \mathrm{~mA}$ for 20 minutes and in the control condition participants received sham stimulation (a current of $2 \mathrm{~mA}$ was applied initially but ramped down to $0 \mathrm{~mA}$ after 30 seconds). As with the WM training, to ensure an equal number of participants in each training condition while maintaining randomization, group assignment was conducted using a randomly shuffled list. The tDCS arm of this study was double-blind, i.e. both participants and researchers were blind to which of the stimulation devices was programmed to administer active and sham stimulation.

\subsubsection{Stimulation Procedure}

Participants received stimulation on their second visit to the laboratory after completing a short re-screening for safety purposes. To maximize comparability with the working memory training, the second visit was always at least 14 days, and no more than 21 days, after the first visit. Immediately following stimulation, participants completed the visual search and monitoring task and the remaining computerized tasks in the standard order. Figure 9 shows the overall set of procedures.

\section{Results at Baseline}

The baseline results were analyzed with a view to replicating previously established task performance and linking individual differences in WMC and IU with behavioral performance and eye movements. At the same time, the effect of increased target 


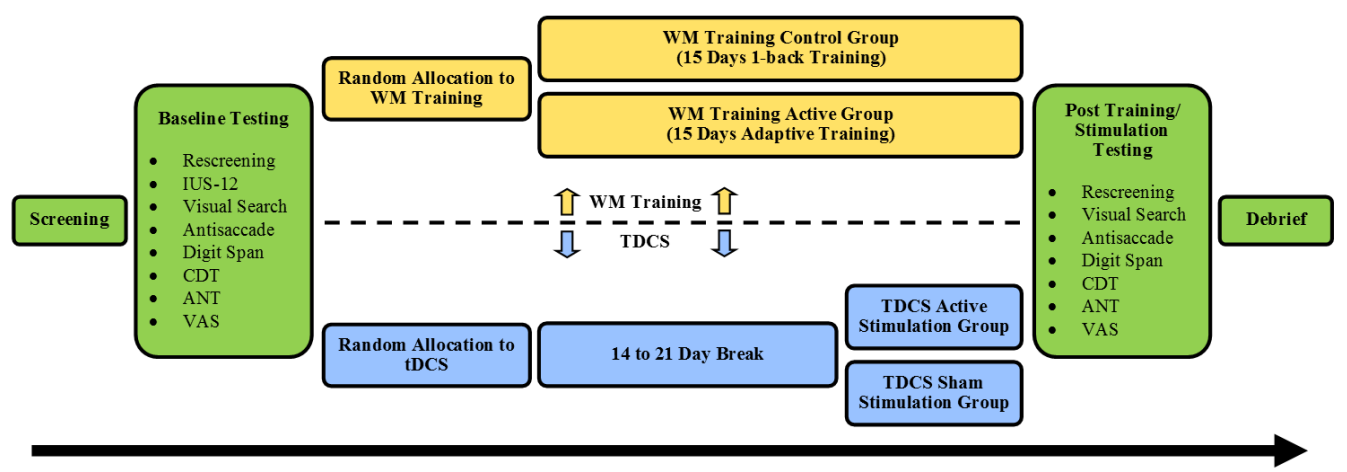

Figure 9. Full study procedure, including the Intolerance of Uncertainty Short Form (IUS-12), the search and monitoring task, the antisaccade task, the digit span, the change detection task (CDT), the Attention Network Test (ANT) and the visual analogue scales (VAS) across the working memory (WM) training and transcranial direct-current stimulation (tDCS) procedures.

prevalence, relative to Muhl-Richardson et al. (2018), were assessed via comparisons with behavioral and first fixation measures from the previous dataset. As all baseline data were collected according to the same procedure all 46 participants were analyzed together. The datasets analyzed in the following sections are available from the corresponding author on reasonable request.

\subsection{Transformations and Exclusions}

Across all analyses (including post-training/stimulation), RT exclusion criteria were set a priori at above $10,000 \mathrm{~ms}$ and below $200 \mathrm{~ms}$ from target onset, but no responses were outside of these limits. RTs were log transformed before being analyzed and untransformed means are reported. In the eye movement data, fixations were excluded from duration analysis if they were longer than $1,200 \mathrm{~ms}$, shorter than $80 \mathrm{~ms}$ in duration, or if they corresponded with a manual response (6.31\% of all fixations). Proportional data were arcsine square root transformed before being analyzed, and untransformed means are reported. Hit rate was defined as the proportion of targets across all trials that were responded to correctly. False alarm rate was defined as the proportion of all responses that were made to non-target stimuli $^{2}$.

\subsection{Behavioral Responses}

Table 1 shows the hit rate, response time (RT) and false alarm rate at baseline, as well as comparisons with previous results from Experiment 3 in Muhl-Richardson et al. (2018). This previous study employed two prevalence levels that were both lower than the present study (blocks where either $5.6 \%$ or $66 \%$ of trials contained at least two targets), therefore, for the purposes of this analysis, the prior data were averaged across prevalence levels. The results show the higher target prevalence level in the present task increased hit rate and reduced RTs and FA rate relative to the previous study.

\footnotetext{
${ }^{2}$ As in Muhl-Richardson et al. (2018), we do not calculate signal detection theory measures of sensitivity or response criterion due to the non-standard calculation of false alarm rate and, given the dynamically changing
} 
Table 1. Mean Basic Behavioral Results from the Present Study at Baseline and Muhl-Richardson et al. (2018) with Unequal Variances t-tests.

\begin{tabular}{lccc}
\hline & $\begin{array}{c}\text { Muhl-Richardson et al. } \\
\text { Experiment } 3\end{array}$ & $\begin{array}{c}\text { Present Study } \\
\text { Baseline }\end{array}$ & \\
\hline Hit Rate & $0.88(0.09)$ & $0.94(0.04)$ & $\mathrm{t}(40.47)=3.65, \mathrm{p}<.001$ \\
Response Time (ms) & $2,685(564)$ & $1,860(351)$ & $\mathrm{t}(49.55)=7.43, \mathrm{p}<.001$ \\
False Alarm Rate & $0.22(0.21)$ & $0.08(0.08)$ & $\mathrm{t}(38.79)=3.72, \mathrm{p}<.001$ \\
\hline
\end{tabular}

Note. Means with standard deviations in parentheses.

\subsection{Target Prediction}

In line with previous work, distractors that were forthcoming targets are referred to as target predictive distractors (TPDs). To examine target prediction, first fixations to targets and TPDs were analyzed to provide a measure of the state of items when they initially attracted attention. These data were analyzed using a one-way (color step: target, TPD one step from becoming a target, TPD two steps from becoming a target) repeated-measures ANOVA. Mauchly's Test of Sphericity indicated that the assumption of sphericity was violated, $\mathrm{W}=0.51, \mathrm{p}<.001$, so Greenhouse-Geisser corrected results are reported. There was a significant effect of color step on the proportion of first fixations to targets and forthcoming targets, $\mathrm{F}(1.35,60.62)=207.60, \mathrm{p}<.001, \eta_{G}^{2}$ $=0.82$. Pairwise comparisons revealed a significant difference between the proportion of first fixations made to targets and TPDs one step from becoming a target, $\mathrm{t}(45)=$ $4.84, \mathrm{p}<.001$, targets and TPDs two steps from becoming a target, $\mathrm{t}(45)=13.51, \mathrm{p}$ $<.001$, and TPDs one and two steps from becoming a target, $\mathrm{t}(45)=32.40, \mathrm{p}<.001$ (see Figure 10). Analysis of fixations and re-fixations to targets and TPDs showed a means of $4.07(\mathrm{SD}=0.55), 4.20(\mathrm{SD}=0.66)$ and $2.93(\mathrm{SD}=0.56)$ total fixations to targets, TPDs one step from becoming a target and TPDs two steps from becoming a target respectively. Background distractors were fixated on average 0.35 times (SD $=0.08)$.

To examine the effects of target prevalence, the proportions of first fixations to forthcoming targets as targets, TPDs one step from becoming a target and TPDs two steps from becoming a target, were compared to averaged results from MuhlRichardson et al. (2018). Three unequal variances t-test were carried out for the data for targets, TPDs one step from becoming a target and TPDs two steps from becoming a target (see Figure 6). In the previous study: (1) a significantly greater proportion of targets were first fixated as targets post-onset $(\mathrm{M}=0.48, \mathrm{SD}=0.22)$, compared to the present study $(\mathrm{M}=0.37, \mathrm{SD}=0.15), \mathrm{t}(54.82)=3.06, \mathrm{p}=.003 ;(2)$ a significantly lower proportion were first fixated at TPDs one step from becoming a target $(\mathrm{M}=$ $0.43, \mathrm{SD}=0.19)$, compared to the present study $(\mathrm{M}=0.53, \mathrm{SD}=0.13), \mathrm{t}(50.12)$ $=3.35, \mathrm{p}=.002$; and $(3)$ there was no difference in the proportion first fixated as TPDs two steps from becoming a target $(\mathrm{M}=0.07, \mathrm{SD}=0.07)$, when compared to the present study $(\mathrm{M}=0.07, \mathrm{SD}=0.05), \mathrm{t}(57.46)=0.74, \mathrm{p}=0.46$. The higher target prevalence used in this study relative to Muhl-Richardson et al. (2018) led to a greater proportion of targets were detected predictively following first fixation one color step prior to onset.

nature of the displays, there is no clear determination of what should constitute a correct rejection. 


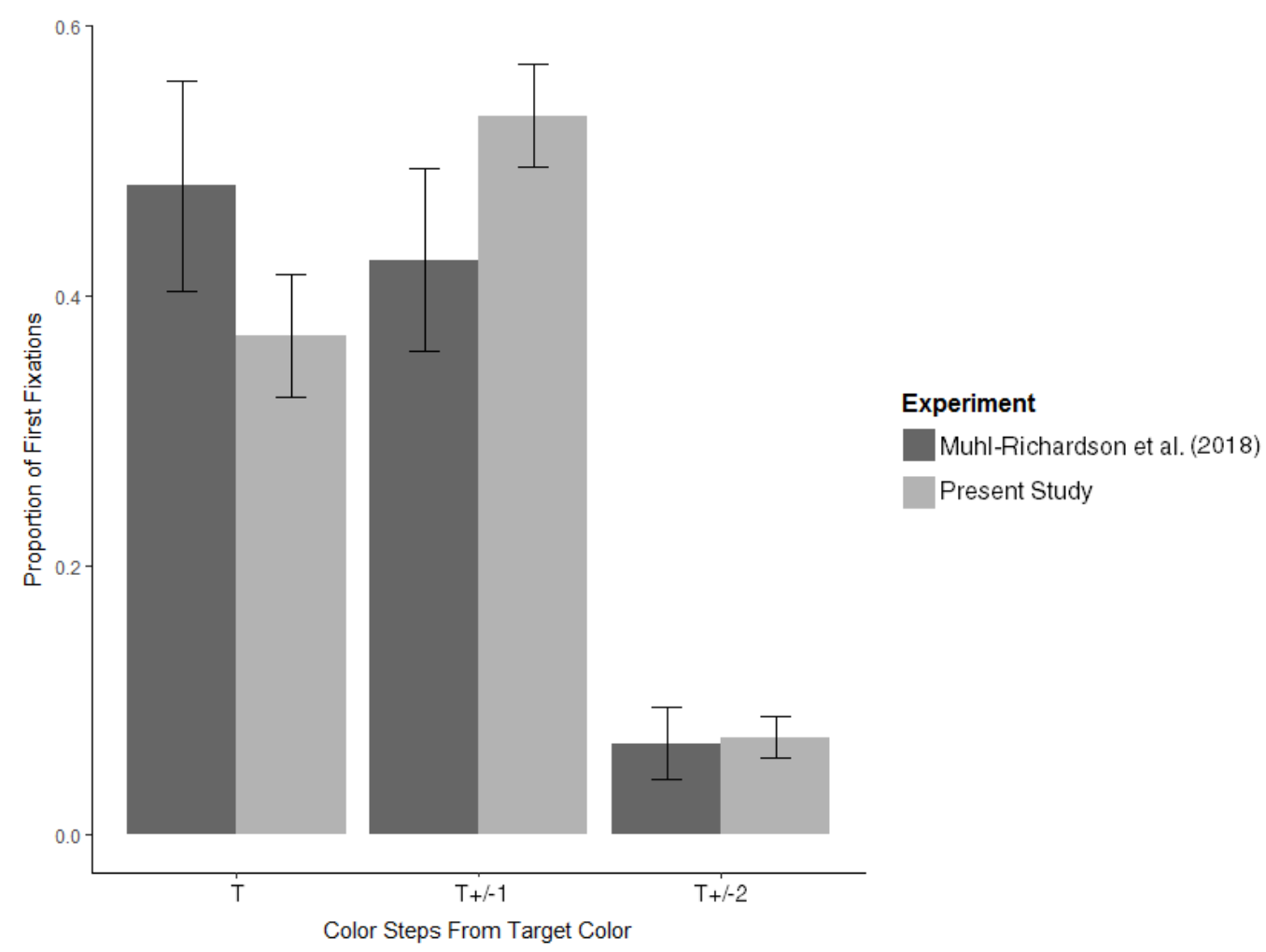

Figure 10. Proportion of first fixations to targets and TPDs one and two steps from becoming targets in both the present study and Muhl-Richardson et al. (2018).

Next, the effects of predictive monitoring on hit rate, RTs and false alarm rate were analyzed. Hit rate was lower when first fixating a TPD one step from becoming a target $(\mathrm{M}=0.93, \mathrm{SD}=0.05)$, than when first fixating the target itself $(\mathrm{M}=0.96$, $\mathrm{SD}=0.04), \mathrm{t}(45)=5.06, \mathrm{p}<.001$. The false alarm rate was not significantly different when fixating a TPD one step from becoming a target $(\mathrm{M}=0.12 \mathrm{SD}=0.12)$ from when first fixating a TPD two steps from becoming a target $(\mathrm{M}=0.13, \mathrm{SD}=0.16)$, $\mathrm{t}(45)=0.71, \mathrm{p}=.481$. RT was significantly lower when first fixating a TPD one step from becoming a target $(\mathrm{M}=1,797 \mathrm{~ms}, \mathrm{SD}=363)$ than when first fixating the target itself $(\mathrm{M}=1,984 \mathrm{~ms}, \mathrm{SD}=383), \mathrm{t}(45)=4.51, \mathrm{p}<.001$.

To summarize these basic behavioral and first fixation results, the increased prevalence rate used in the present study increased hit rate, and lowered RT and false alarm rate, relative to the prevalence level used in Muhl-Richardson et al (2018). The pattern of first fixations to targets and forthcoming targets was different across studies. In the present study, more targets were first fixated one step before onset at the target color and fewer were first fixated post-onset at the target color compared to Muhl-Richardson et al. (2018), suggesting an effect of target prevalence upon search guidance. Furthermore, in the present study, targets first fixated one step before reaching the target color received significantly faster post-onset responses than those targets first fixated post-onset. 


\subsection{Individual Differences at Baseline}

The mean score on the IUS-12 was $27.20(\mathrm{SD}=7.92)$ and the mean visual digit span score was $7.04(\mathrm{SD}=1.62)$. While no comparison can be made for the digit span (due to using a different task), the IUS-12 data was similar to that observed in a previous study $(\mathrm{M}=28.72, \mathrm{SD}=9.96), \mathrm{t}(56.69)=0.63, \mathrm{p}=.53$ (Muhl-Richardson et al., 2018). LMM analyses were performed in $\mathrm{R}$ (v3.4.0) using the lme4 package (v1.1-7; Bates, Mchler, Bolker, Walker, 2014). These models allow analysis of these data and their interaction as continuous variables and, because they do not rely upon aggregated means, are more robust to missing data and outliers. All models tested included participant as a random factor. The aim of this analysis was to replicate previous findings linking IU and verbal WMC to behavioral performance and eye movements and, as such, model fitting began in all cases with a model containing the two-way interaction between IU and verbal WMC and iterated through more parsimonious variants until reaching the best-fitting model (any models that failed to converge were excluded). The effects of individual differences in verbal WMC and IU were considered in relation to a number of behavioral and eye movement measures. RT, fixation duration, number of fixations per trial and scanpath length were all analyzed using standard LMMs. Hit rate, false alarm rate and an index of the extent predictive fixations to TPDs (calculated as the rate of first fixation of targets as TPDs one step from becoming a target compared to as targets post-onset) were analyzed as binary variables using binomial generalized linear mixed models (GLMMs). See Table 2 for LMM results. The coefficients shown for the GLMM on hit rate in Table 2 are, as is standard for binomial GLMMs, in logit units. For the effect of IU on hit rate, the coefficient of 0.04 corresponds to an increase in hit rate of 0.07 across the range of observed IU scores. The IU effect, and its interaction with verbal WMC, is also shown in Figure 11. In summary, IU was found to be positively associated with hit rate and this effect was moderated by verbal WMC, such that the association between IU and hit rate was strongest in individuals with high verbal WMC.

Table 2. GLMMs and LMMs Examining Individual Differences in Verbal WMC and IU at Baseline.

\begin{tabular}{|c|c|c|c|c|c|c|c|}
\hline & Hit Rate & $\begin{array}{l}\text { Response } \\
\text { Time }\end{array}$ & $\begin{array}{c}\text { False Alarm } \\
\text { Rate }\end{array}$ & $\begin{array}{l}\text { Color Step From } \\
\text { T At First Fixation }\end{array}$ & $\begin{array}{l}\text { Scanpath } \\
\text { Length }\end{array}$ & $\begin{array}{l}\text { Number of } \\
\text { Fixations }\end{array}$ & $\begin{array}{l}\text { Fixation } \\
\text { Duration }\end{array}$ \\
\hline Constant & $\begin{array}{l}3.02^{*} \\
(0.12)\end{array}$ & $\begin{array}{l}7.36^{*} \\
(0.03)\end{array}$ & $\begin{array}{c}-2.92^{*} \\
(0.17)\end{array}$ & $\begin{array}{l}0.39^{*} \\
(0.09)\end{array}$ & $\begin{array}{l}782.40^{*} \\
(18.92)\end{array}$ & $\begin{array}{c}126.97^{*} \\
(2.27)\end{array}$ & $\begin{array}{l}5.46^{*} \\
(0.03)\end{array}$ \\
\hline IU & $\begin{array}{c}0.04^{*} \\
(0.02)\end{array}$ & & & & & & \\
\hline Verbal WMC & $\begin{array}{l}-0.04 \\
(0.07)\end{array}$ & & & & & & \\
\hline IU x Verbal WMC & $\begin{array}{c}0.02^{*} \\
(0.01)\end{array}$ & & & & & & \\
\hline Observations & 7,597 & 7,176 & 7,876 & 6,873 & 3,312 & 3,312 & 3,312 \\
\hline Log Likelihood & $-1,578.79$ & $-5,830.39$ & $-2,002.89$ & $-4,491.52$ & $-20,728.40$ & $-13,697.47$ & $3,526.15$ \\
\hline Akaike Inf. Crit. & $3,167.58$ & $11,666.79$ & $4,009.79$ & $8,987.03$ & $41,462.80$ & $27,400.94$ & $-7,046.30$ \\
\hline Bayesian Inf. Crit. & $3,202.26$ & $11,687.42$ & $4,023.73$ & $9,000.71$ & $41,481.11$ & $27,419.26$ & $-7,027.99$ \\
\hline
\end{tabular}




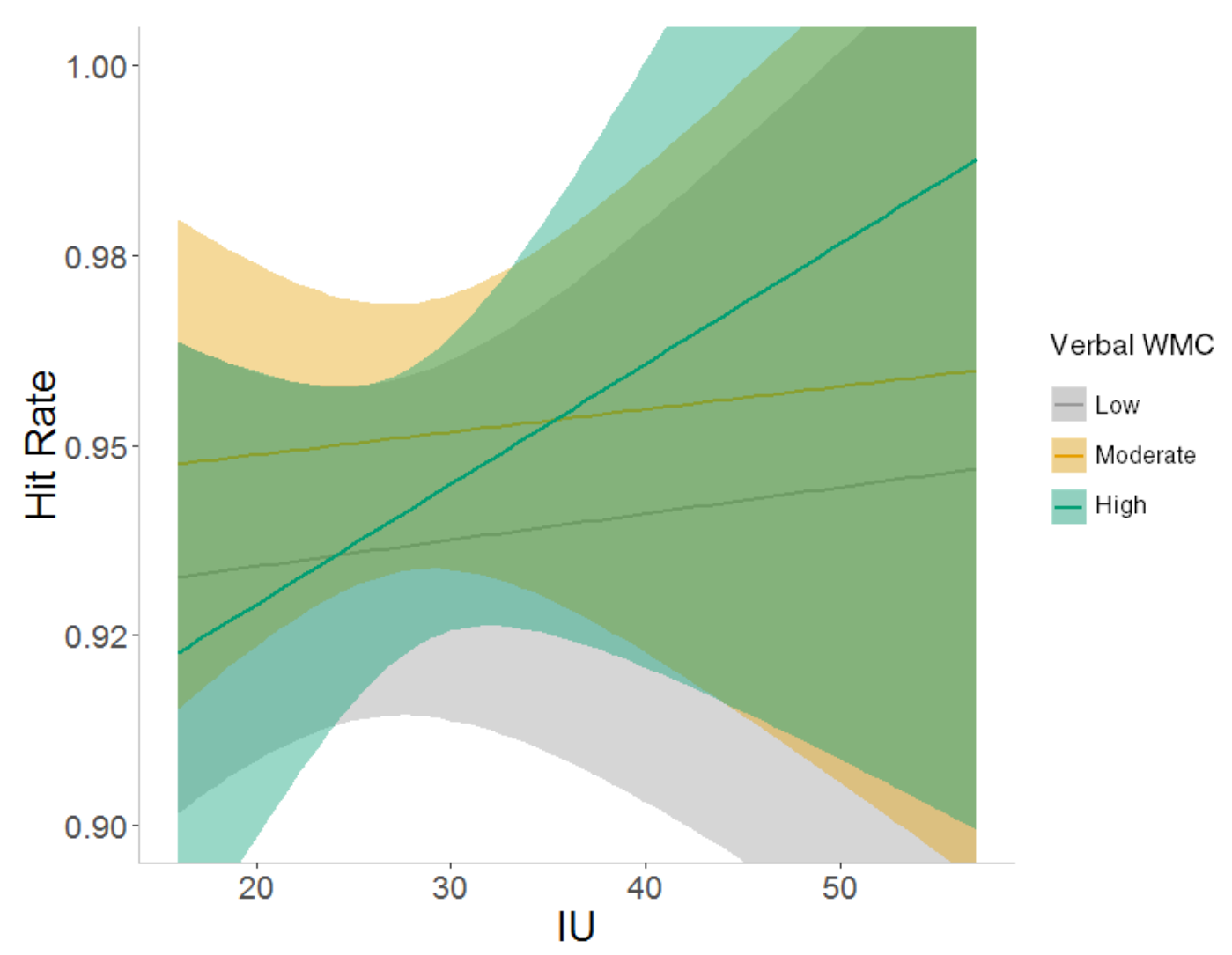

Figure 11. Hit rate and intolerance of uncertainty (IU) at low, moderate and high levels of verbal working memory capacity (WMC; shaded regions show continuous 95\% CIs). Verbal WMC was split at the 33rd and 66 th percentiles to give three categories. This categorization was only for visualization and these data were included in all analyses as continuous data.

\section{Working Memory Training Results}

IU and VAS measures were analyzed to check for baseline group and/or time differences. A between-subjects t-test indicated that there was no significant difference in IU between the two training groups, $\mathrm{t}(19)=1.34, \mathrm{p}=.197$. An ANOVA was conducted for all VAS and included training group and time as factors. There was a main effect of group on the VAS concentration item, $\mathrm{F}(1,18)=4.74, \mathrm{p}=.043, \eta_{G}^{2}=0.19$, such that those who received active training reported better concentration $(\mathrm{M}=109, \mathrm{SD}=21)$ than those who received control training $(\mathrm{M}=90, \mathrm{SD}=19)$. Similar effects of group on the VAS alert, $\mathrm{F}(1,18)=4.33, \mathrm{p}=.052, \eta_{G}^{2}=0.17$, and happy, $\mathrm{F}(1,18)=4.32, \mathrm{p}=$ $.052, \eta_{G}^{2}=0.17$, items neared significance. Those who received active training tended to be marginally more alert $(\mathrm{M}=97, \mathrm{SD}=28)$ and happier $(\mathrm{M}=104, \mathrm{SD}=20)$ than those who received control training (alert: $\mathrm{M}=73, \mathrm{SD}=25$; happy: $\mathrm{M}=86, \mathrm{SD}=$ $21)$. There were no other significant main effects or interactions of group and time in terms of any other VAS measures, Fs $<=2.94$. These results show that groups did not differ in IU and that there was only a limited group difference in mood. 


\subsection{Working Memory Training Performance}

The training task performance of those who completed the adaptive WM training was considered in terms of the mean n-back level attained during a daily session. Analysis showed improvement in the active WM training group, with a significant increase between first $(\mathrm{M}=2.04, \mathrm{SD}=0.56)$ and final training sessions $(\mathrm{M}=2.83, \mathrm{SD}=$ $0.85), \mathrm{t}(9)=3.42, \mathrm{p}=.008$.

\subsection{Search Task}

Data from the visual search task were analyzed to examine the effects of WM training on basic behavioral and target prediction measures reported at baseline in the previous section. In addition to traditional ANOVA results, Bayes factors from JeffreysZellnerSiow (JZS) Bayesian model comparisons with default prior scales are reported (JASP Team, 2018; Rouder, Haaf, Aust, 2018; Wagenmakers et al., 2018) to evaluate evidence both for and against transfer effects.

There was a significant effect of time upon hit rate, $\mathrm{F}(1,18)=17.82, \mathrm{p}<.001, \eta_{G}^{2}$ $=0.28, \mathrm{RT}, \mathrm{F}(1,18)=68.88, \mathrm{p}<.001, \eta_{G}^{2}=0.23$, and false alarm rate, $\mathrm{F}(1,18)=$ $50.81, \mathrm{p}=.001, \eta_{G}^{2}=0.13$, such that hit rate increased and false alarm rate and RT decreased $^{3}$. There were no significant effects of training group and no significant group by time interactions for either hit rate or RT, Fs $<=2.32$. The interaction between group and time with regard to false alarm rate neared statistical significance, $\mathrm{F}(1,18)$ $=3.67, \mathrm{p}=.065, \eta_{G}^{2}=0.04$. The active training group had a higher false alarm rate pre-training than the control group, but this difference narrowed post-training (means are shown in Table 3).

Further analysis of hit rate revealed that the model including only time as a factor was preferred to the model including the interaction of group and time by a Bayes factor of 4.76. The data provide moderate support against the hypothesis that training group and time interact in their effect on hit rate in the search task. For RT, the model including only time as a factor was preferred to the model including the interaction of group and time by a Bayes factor of 3.89. The data provide moderate support against the hypothesis that training group and time interact in their effect on RT in the search task. For FA rate, the model including the interaction of group and time was preferred to the model including only time as a factor by a Bayes factor of 1.27. The data provide anecdotal support for the hypothesis that training group and time interact in their effect on false alarm in the search task.

To test the effects of WM training upon target prediction, a $2 \times 2 \times 3$ (time $\mathrm{x}$ training group x color step) ANOVA was conducted on the proportion of first fixations made to targets, TPDs at one and two steps from the target color. The purpose of this analysis was to examine the effect of WM training on target prediction so only the two- and three-way interactions between time and color step and time, color step and training group and were examined and none of these interactions were significant, Fs $<=1.07$. Further analysis revealed that the model including only color step as a factor was preferred to the model including the interaction between time and color step by a Bayes factor of 38.87 and to the model including the three-way interaction between time, color step and training group by a Bayes factor of 6,522 . The data provide extreme evidence against the hypothesis that time and training group interact in their effect on target prediction in the search task.

\footnotetext{
${ }^{3}$ Where possible, all such analyses across all tasks were also attempted using difference scores, but the results
} were no different to when time was included as a factor. 
Table 3. Mean Hit Rate, Response Time and False Alarm Rate in the Search Task.

\begin{tabular}{lllll}
\hline & \multicolumn{2}{c}{ Control Training } & \multicolumn{2}{c}{ Active Training } \\
& Pre-training & Post-training & Pre-training & Post-training \\
\hline Hit Rate & $0.94(0.03)$ & $0.97(0.02)$ & $0.93(0.04)$ & $0.97(0.02)$ \\
Response Time $(\mathrm{ms})$ & $1,889(299)$ & $1,592(310)$ & $1,953(410)$ & $1,601(250)$ \\
False Alarm Rate & $0.05(0.03)$ & $0.03(0.03)$ & $0.10(0.09)$ & $0.04(0.04)$ \\
\hline
\end{tabular}

Note. Standard deviations in parentheses.

\subsection{Working Memory Capacity and Attentional Control Tasks}

The effects of time (pre- and post-training) and training group were considered with respect to performance in the digit span, change detection, ANT and antisaccade tasks. If the benefits of active training transfer to untrained tasks, this should be evident in a significant group by time interaction. As above, Bayes factors from JZS Bayesian model comparisons with default prior scales are reported to evaluate evidence both for and against transfer effects.

There were no significant effects of time or group (or interactions) with respect to digit span scores, (Fs $<=2.40$ ). Further analysis revealed that the model including only participant as a factor (null model) was preferred to the model including the interaction of group and time by a Bayes factor of 11.86. The data provide strong support against the hypothesis that training group and time interact in their effect on digit span.

In the $\mathrm{CDT}$, hit rate improved over time $(\mathrm{M}=0.73, \mathrm{SD}=0.45$ to $\mathrm{M}=0.79, \mathrm{SD}$ $=0.41), \mathrm{F}(1,18)=9.42, \mathrm{p}=.007, \eta_{G}^{2}=0.13$, and the effect of time upon RT also neared significance $(\mathrm{M}=744 \mathrm{~ms}, \mathrm{SD}=124$ to $\mathrm{M}=678 \mathrm{~ms}, \mathrm{SD}=116), \mathrm{F}(1,18)=$ $4.00, \mathrm{p}=.061, \eta_{G}^{2}=0.07$. There were no other significant effects or interactions, Fs $<=0.43$. Further analysis of hit rate in the CDT revealed that the model including only the main effect of time was preferred to the model including the interaction of group and time by a Bayes factor of 4.85 . The data provide moderate support against the hypothesis that training group and time interact in their effect on hit rate in the CDT. For RT, the model including time was preferred to the model including the interaction of group and time by a Bayes factor of 4.85. The data provide moderate evidence against the hypothesis that training group and time interact in their effect on RT in the CDT.

There were no significant effects or interactions in terms of accuracy or first saccade latency on antisaccade trials, Fs $<=1.44$. Further analysis of the accuracy data revealed that the model including only participant as a factor (null model) was preferred to the model including the interaction of group and time by a Bayes factor of 13.63. The data provide strong support against the hypothesis that training group and time interact in their effect on hit rate in the antisaccade task. For the first saccade latency data, the model including only participant as a factor (null model) was preferred to the model including the interaction of group and time (and the main effect only of onset delay) by a Bayes factor of 10.78. The data provide strong support against the hypothesis that training group and time interact in their effect on accuracy in the antisaccade task.

In the ANT, the alerting score improved over time from $42.91(\mathrm{SD}=32.31)$ to 87.32 
$(\mathrm{SD}=42.91), \mathrm{F}(1,18)=4.97, \mathrm{p}=.042, \eta_{G}^{2}=0.04$. There were no other significant effects or interactions, Fs $<=2.26$. Further analysis of the ANT orienting network data revealed that the model including only participant as a factor (null model) was preferred to the model including the interaction of group and time by a Bayes factor of 17.05. The data provide strong support against the hypothesis that training group and time interact in their effect on ANT orienting network score. For the ANT alerting network, the model including only time as a factor was preferred to the model including the interaction of group and time by a Bayes factor of 4.73 . The data provide moderate support against the hypothesis that training group and time interact in their effect on ANT alerting network score. For the ANT executive control network, the model including only participant as a factor (null model) was preferred to the model including the interaction of group and time by a Bayes factor of 2.58. The data provide anecdotal support against the hypothesis that training group and time interact in their effect on ANT executive control network score.

\subsection{Summary of Working Memory Training Results}

In summary, while there was some improvement in task performance post-training (hit rate increased and FA and RT rates decreased over time), there was no evidence that WM training differentially impacted on performance in the visual search task and no evidence that the effects of WM training generalized beyond performance in the n-back itself. Indeed, the Bayes factors for all but one result demonstrate that the data provide support against the hypothesis that training group and time interact (i.e. against the prediction that there was a benefit of active training) and indicate that the absence of this result was not due to a lack of power. Given these results, no examination of individual differences was conducted at the level of the training subgroups.

\section{Transcranial Direct-current Stimulation Results}

IU and VAS measures were analyzed to check for baseline group and/or time differences. A between-subjects t-test indicated that there was no significant difference in IU between the two stimulation groups, $\mathrm{t}(25)=0.03, \mathrm{p}=.979$. An ANOVA was conducted for all VAS and included stimulation group and time as factors. There was a group by time interaction for the VAS item that asked participants to rate how hard they tried, $\mathrm{F}(1,23)=8.36, \mathrm{p}=.008, \eta_{G}^{2}=0.07$. In the active stimulation group, participants reported trying less hard during their first visit $(\mathrm{M}=111, \mathrm{SD}=22)$, than during their second visit $(\mathrm{M}=125, \mathrm{SD}=11), \mathrm{t}(12)=3.21, \mathrm{p}=.007$. No difference was observed in the sham stimulation group, $\mathrm{t}(11)=0.78, \mathrm{p}=.455$. There were no other significant main effects or interactions of group and time in terms of any other VAS measures, $F<=3.83$. These results show that groups did not differ in IU and there was no clear evidence of a difference in effort (beyond that attributable to active stimulation itself).

\subsection{Search Task}

As for the WM training results, Bayes factors from JZS Bayesian model comparisons with default prior scales are reported to evaluate evidence both for and against stim- 
ulation effects. There was a significant effect of time upon hit rate, $\mathrm{F}(24)=11.49$, $\mathrm{p}=.002, \eta_{G}^{2}=0.12, \mathrm{RT}, \mathrm{F}(24)=22.09, \mathrm{p}<.001, \eta_{G}^{2}=0.10$, and false alarm rate, $\mathrm{F}(24)=13, \mathrm{p}=.009, \eta_{G}^{2}=0.13$, such that all three reflected improved performance post-stimulation relative to baseline. There were no significant effects of stimulation group and no significant group by time interactions, Fs $<=1.23$. Means are shown in Table 4. Further analysis of hit rate revealed that the model including only time as a factor was preferred to the model including the interaction of group and time by a Bayes factor of 4.34 . The data provide moderate support against the hypothesis that stimulation group and time interact in their effect on hit rate in the search task. For RT, the model including time and group as non-interacting factors was preferred to the model including the interaction of group and time by a Bayes factor of 2.36. The data provide anecdotal support against the hypothesis that stimulation group and time interact in their effect on RT in the search task. For FA rate, the model including only time as a factor was preferred to the model including the interaction of group and time by a Bayes factor of 5.67. The data provide moderate support against the hypothesis that stimulation group and time interact in their effect on false alarm in the search task.

A $2 \times 3 \times 3$ (time $\mathrm{x}$ stimulation group $\mathrm{x}$ color step) ANOVA was carried out, this time to assess the impact of tDCS upon target prediction. Again, only the two- and threeway interactions between time and color step and time, color step and stimulation group and were examined and none of these interactions were significant, Fs $<=0.98$. The pattern of data did not differ significantly from that shown in Figure 5.

Further analysis revealed that the model including the interaction between color step and stimulation group was preferred to the model including the interaction between time and color step by a Bayes factor of $1.85 \times 10^{5}$ and to the model including the three-way interaction between time, color step and stimulation group by a Bayes factor of 691 . The data provide extreme evidence against the hypothesis that time and stimulation group interact in their effect on target prediction in the search task.

Table 4. Mean Hit Rate, Response Time (RT) and False Alarm Rate in the Search Task by Stimulation Group and Time

\begin{tabular}{lcccc}
\hline & \multicolumn{2}{c}{$\begin{array}{c}\text { Sham Stimulation } \\
\text { Pre-stimulation }\end{array}$} & $\begin{array}{c}\text { Post-stimulation } \\
\text { Pre-stimulation Stimulation }\end{array}$ & Post-stimulation \\
\hline Hit Rate & $0.94(0.03)$ & $0.97(0.03)$ & $0.96(0.04)$ & $0.98(0.01)$ \\
Response Time (ms) & $1,934(387)$ & $1,670(353)$ & $1,691(274)$ & $1,516(236)$ \\
False Alarm Rate & $0.07(0.06)$ & $0.04(0.03)$ & $0.09(0.11)$ & $0.03(0.03)$ \\
\hline
\end{tabular}

Note. Standard deviations in parentheses.

\subsection{Working Memory Capacity and Attentional Control Tasks}

The effects of time (pre- and post-stimulation) and training group were considered with regard performance in the digit span, change detection, ANT and ant saccade tasks. Similarly to the effects of WM training, if there were benefits of active, relative to sham, stimulation generalize to these task this should be evident in a significant group by time interaction. Again, Bayes factors from JZS Bayesian model comparisons with default prior scales are once more reported to evaluate evidence both for and against 
stimulation effects.

Digit span improved over time from $6.85(\mathrm{SD}=1.62)$ to $7.42(\mathrm{SD}=1.42), \mathrm{F}(1,24)$ $=9.26, \mathrm{p}=.006, \eta_{G}^{2}=0.02$, but there was no significant effect of group and no significant interaction with time, Fs $<=0.38$. Further analysis revealed that the model including main effects of time and the span direction was preferred to a model that also included the interaction between stimulation group and time by a Bayes factor of 7.46. The data provide moderate evidence against the hypothesis that time and stimulation group interact in their effect on digit span.

In the change detection task there were significant improvements in hit rate over time, $\mathrm{F}(1,24)=32.02, \mathrm{p}<.001, \eta_{G}^{2}=0.07$, and $\mathrm{RT}, \mathrm{F}(1,24)=8.90, \mathrm{p}=.006, \eta_{G}^{2}=$ 0.08 . There was also a significant interaction between group and time in their effect on hit rate in this task, $\mathrm{F}(1,24)=4.59, \mathrm{p}=.042, \eta_{G}^{2}=0.01$. Pairwise comparisons revealed that both the control group, $\mathrm{t}(12)=5.84, \mathrm{p}<.001$, and the experimental group, $\mathrm{t}(12)$ $=2.36, \mathrm{p}=.036$, improved over time. Further analysis of the hit rate data revealed that the model including the interaction of group and time was preferred to the model including only the main effect of time by a Bayes factor of 1.03. The data provide anecdotal evidence for the hypothesis that stimulation group and time interact in their effect on hit rate in the CDT. The means (see Table 4) show that this interaction was driven by a greater improvement over time in the sham stimulation group relative to the active group, indicating that this interaction does not represent a benefit of active stimulation. For the RT data, the model including time was preferred to the model including the interaction of group and time by a Bayes factor of 3.26. The data provide moderate evidence against the hypothesis that stimulation group and time interact in their effect on RT in the CDT.

There were no significant effects or interactions in terms of accuracy or first saccade latency on antisaccade trials in the antisaccade task, Fs $<=1.43$. Further analysis of the accuracy data revealed that the model including only participant as a factor (null model) was preferred to the model including the interaction of group and time by a Bayes factor of 1.62. The data provide anecdotal support against the hypothesis that stimulation group and time interact in their effect on hit rate in the antisaccade task. For first saccade latency, the model including only participant as a factor (null model) was preferred to the model including the interaction of group and time by a Bayes factor of 28.18. The data provide strong support against the hypothesis that stimulation group and time interact in their effect on hit rate in the antisaccade task.

There were also no significant effects or interactions in terms of the altering, orienting or executive control networks in the ANT, Fs $<=1.86$. Further analysis of the ANT orienting network data revealed that the model including only participant as a factor (null model) was preferred to the model including the interaction of group and time by a Bayes factor of 7.90. The data provide moderate support against the hypothesis that stimulation group and time interact in their effect on ANT orienting network score. For the ANT alerting network data, the model including only participant as a factor (null model) was preferred to the model including the interaction of group and time by a Bayes factor of 20.19. The data provide moderate support against the hypothesis that stimulation group and time interact in their effect on ANT alerting network score. For the ANT executive control network data, the model including only participant as a factor (null model) was preferred to the model including the interaction of group and time by a Bayes factor of 23.37. The data provide moderate support against the hypothesis that stimulation group and time interact in their effect on ANT executive control network score. 
Table 5. Mean Hit Rate, Response Time (RT) and False Alarm Rate in the Search Task by Stimulation Group and Time

\begin{tabular}{lcccc}
\hline & \multicolumn{2}{c}{ Sham Stimulation } & \multicolumn{2}{c}{ Active Stimulation } \\
& Pre-stimulation & Post-stimulation & Pre-stimulation & Post-stimulation \\
\hline Hit Rate & $0.69(0.10)$ & $0.77(0.08)$ & $0.72(0.14)$ & $0.76(0.11)$ \\
Response Time (ms) & $815(164)$ & $714(121)$ & $760(119)$ & $708(145)$ \\
\hline
\end{tabular}

Note. Standard deviations in parentheses.

\subsection{Summary of Transcranial Direct-current Stimulation Results}

In summary, there was no indication that active anodal tDCS of the left DLPFC provided a specific benefit relative to sham stimulation in the search and monitoring task, or indeed any of the other tasks participants performed. Performance tended to improve over time on most tasks, but the Bayes factors demonstrate that overall the data provide support against the hypothesis that stimulation group and time interact (against the prediction that there was a benefit of active stimulation) and indicate that the absence of this result was not due to a lack of power. Again, given these results, no examination of individual differences was conducted at the level of the stimulation subgroups.

\section{Follow-up Individual Differences Results}

Our previous analyses suggest that the only effect present in WM and tDCS procedures was that of practice, given these results, we decided to analyse the post-WM training/tDCS data as a reliability check for further evidence of the link between IU and verbal WMC and target detection in the visual search task. For this analysis, we focused specifically on the visual search task, IU and verbal WMC data across both arms and time points of the study. The earlier GLMM analysis of IU, verbal WMC and hit rate in the visual search task was repeated including time as a factor (see Table 6). This analysis supports the previous analysis performed at baseline. Consistent with practice effects and earlier ANOVA analyses there was a significant overall effect of time. More interestingly, IU positively predicted hit rate independently of time. The interaction between IU and verbal WMC followed the same pattern as was previously found, and only narrowly fell outside the bounds of statistical significance (see Figure 12). The interaction between IU and time also narrowly missed statistical significance, such that IU tended to predict hit rate less strongly at the second time point. These final results are consistent with a practice effect that enables individuals to overcome some of the difficulties associated with IU given sufficient practice.

\section{General Discussion}

The present study focuses on a novel dynamic visual search task that requires participants to monitor a large array of dynamically changing colored squares over time. This task was designed to simulate a range of real-world visual monitoring tasks involving complex electronic displays of information, such as marine radar and geological imag- 


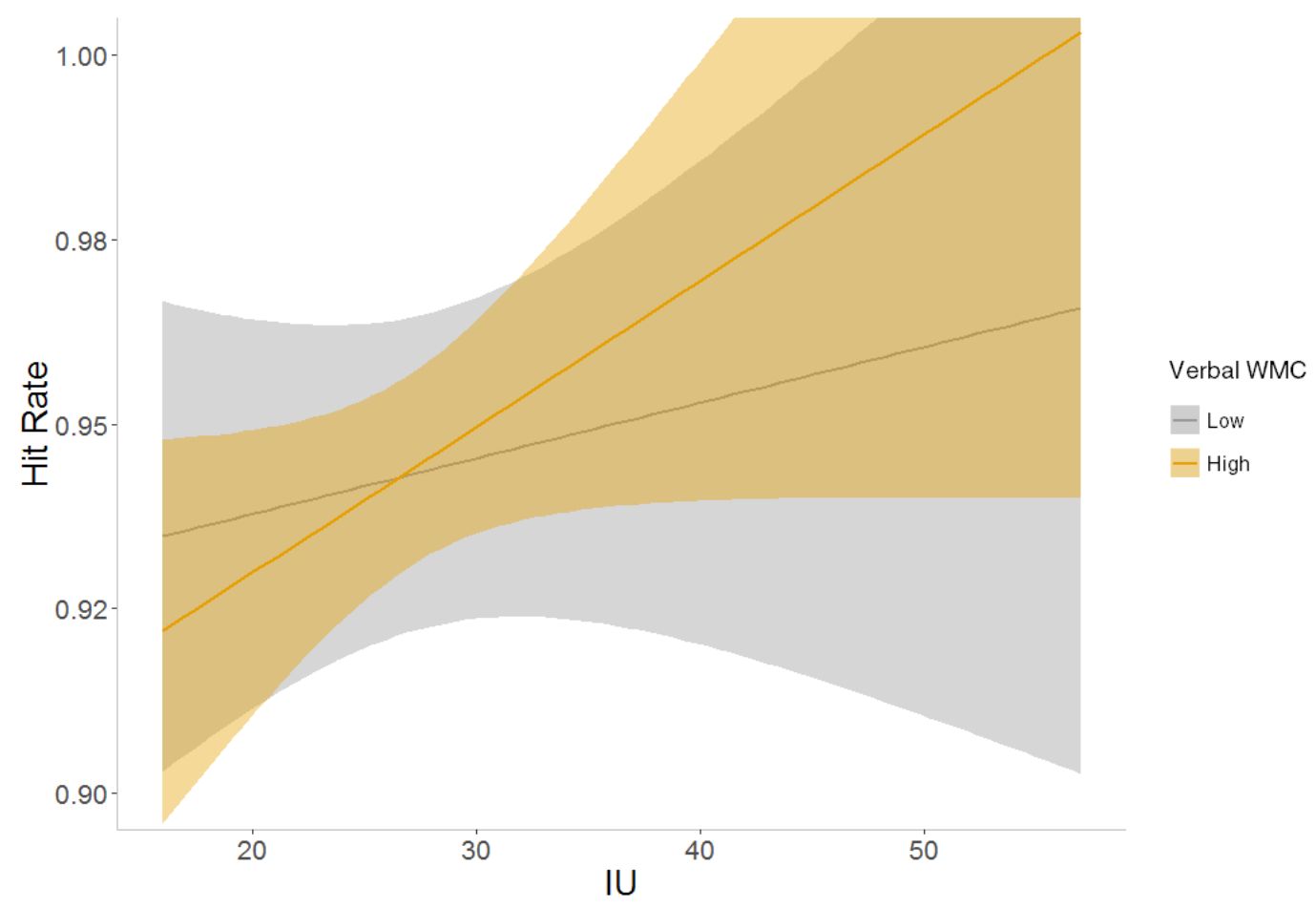

Figure 12. Hit rate and intolerance of uncertainty (IU) at low and high levels of verbal working memory capacity (WMC; shaded regions show continuous 95\% CIs). Verbal WMC was split at the median to give two categories. This categorization was only for visualization and these data were included in all analyses as continuous data.

ing. The primary goal of the present study was to assess the efficacy of WM training and tDCS of the left DLPFC in improving performance in a dynamic search task, but we also sought to replicate and extend previous findings relating to target detection and prediction in conditions of high target prevalence (Muhl-Richardson et al., 2018) and to confirm the influence of IU and verbal WMC in predicting false alarm rate and eye movements in the same dynamic search task.

To replicate and extend previous findings, we began by examining basic performance and eye movements from the search and monitoring task. The results showed that the higher level of target prevalence in the present study led to a higher hit rate, faster RTs and a lower false alarm rate compared with a previous study of the same task where target prevalence was low (Muhl-Richardson et al., 2018). These differences in behavioral performance are consistent with the well-established effect of target prevalence on the criterion used for the perceptual identification of targets (Godwin et al., 2014). The present study also found that the higher level of target prevalence in the present study increased predictive first fixations to TPDs and supported faster RTs for targets first fixated predictively. This supports the account that high target prevalence in the present class of dynamic task increases the validity of TPDs as cues to target onsets. These results suggest that a novel prevalence effect in search and monitoring tasks like this one is to impact the utility of target-predictive stimuli. In low prevalence conditions, such stimuli are often examined, but afford no RT benefit, while in high prevalence conditions, examining these stimuli helps to speed RTs. 
Table 6. GLMM Examining Individual Differences in Verbal WMC and IU and Hit Rate Within the Overall Pooled Dataset at Both Time Points.

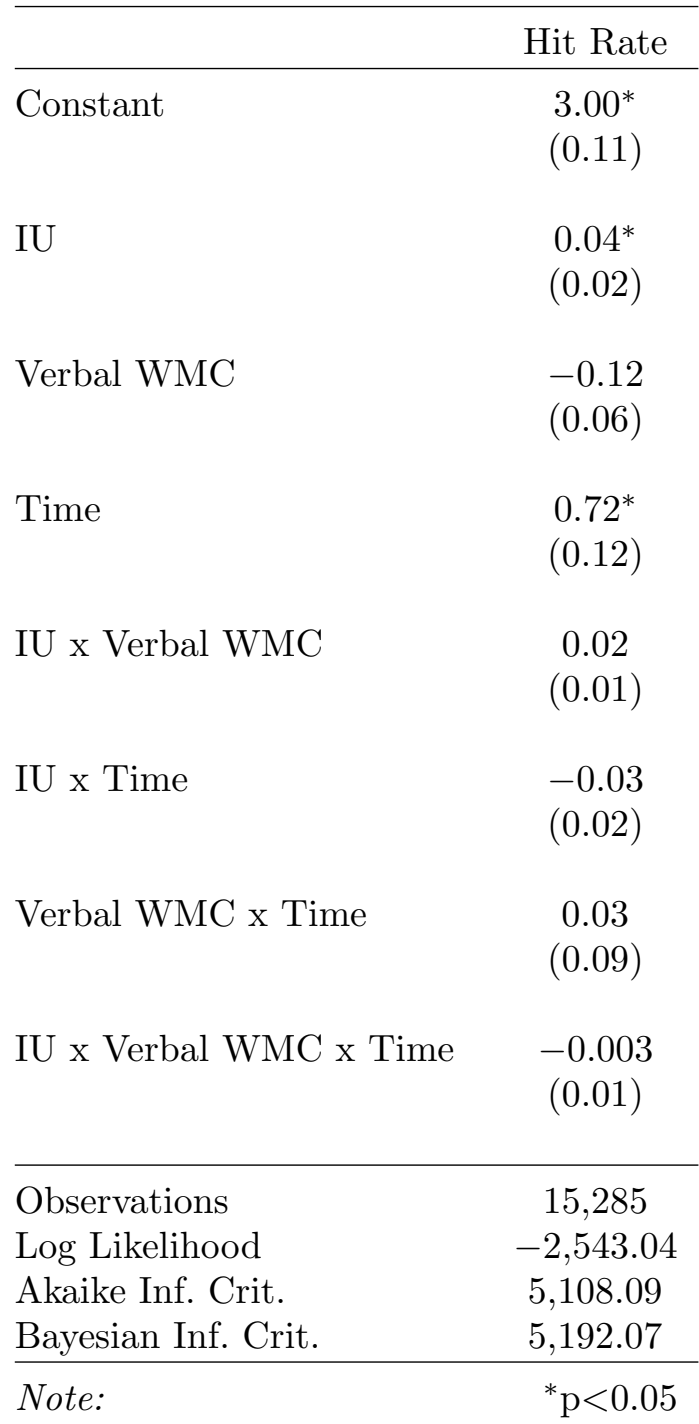

The present study also aimed confirm the previously observed roles of IU and verbal WMC as performance predictors in the search and monitoring task. A Signal Detection Theory (SDT) analysis was not possible in the present case, however, SDT provides a clear theoretical framework that can be used to conceptualize these results and relevant terminology is used accordingly. IU was found to be positively associated with hit rate in the search task and this effect was moderated by verbal WMC, such that the association between IU and hit rate was strongest in individuals with high verbal WMC. In contrast, a previous study in which target prevalence was lower showed that individuals who reported increased symptoms of IU were more likely to identify non-targets as targets (there was a positive association with false alarm rate) and this relationship was moderated by verbal WMC, such that it was strongest in individuals with low verbal WMC. The shift in the effect of IU (and its interaction with verbal WMC) from false alarm rate to hit rate can be explained in terms of an association between IU and response criterion.

In both the present and previous studies, the findings indicate that WMC is impor- 
tant in mediating the influence of IU on task performance. In Muhl-Richardson et al. (2018), the cost of high IU to false alarm rate was driven primarily by individuals with low verbal WMC and, similarly, in the present study, the benefit of high IU to hit rate was driven primarily by individuals with high verbal WMC. While our design did not support a standard SDT analysis, it is still useful to consider our results within this framework. These results are consistent with an effect of verbal WMC on perceptual sensitivity. An increase in sensitivity, leads to targets being more reliably discriminated from distractors (and could improve hit rate; i.e. if the signal distribution was further separated from the noise distribution). Similarly, a decrease in sensitivity, associated with low verbal WMC, would mean that targets and distractors were less easily discriminated, and false alarm rate could be increased (i.e. if the noise distribution overlapped more with the signal distribution). This account is illustrated in Figure 13 using hypothetical signal and noise distributions that are consistent with our findings. Figure 13a illustrates a criterion shift associated with IU and Figure 13b combines this with a change in perceptual sensitivity associated with verbal WMC.

The account we provide is supported by previous literature which links WM to perceptual sensitivity. For example, one study found that WMC mediated the effects of externalizing mental health disorders on sensitivity in a go/no-go learning task (Endres, Rickert, Bogg, Lucas, Finn, 2011) and another found that high WMC individuals demonstrated greater sensitivity than low WMC individuals when judging time intervals (Broadway Engle, 2011). Of particular note is Unsworth and Engles (2007) framework for individual differences in WMC, which states that low WMC individuals typically experience greater interference from irrelevant information due to a reduced ability to discriminate this from relevant information and an increased reliance upon external cues to guide attention. Furthermore, a series of perceptual identification experiments demonstrated that cues held in WM significantly improved sensitivity (Soto, Wriglesworth, Bahrami-Balani, Humphreys, 2010). They also found that verbal cues were as effective as visual cues and suggest that the conceptual match between a verbal cue and a visual target is sufficient for this boost to sensitivity. In terms of the present study, this is consistent with an account where participants used self-assigned verbal labels to improve their discrimination of targets from target-similar colors.

The present study examined the effects of both a program of dual n-back WM training and tDCS of the DLPFC upon performance in the complex search task and, to check for a range of other near transfer effects, a set of WM and attention tasks. Improvements in the active training and stimulation groups (relative to controls) were predicted across all tasks, but Bayesian analysis showed that the data provided support against active training and stimulation effects. Our analysis indicates that this result was not due to a lack of power, but there are still a number of possible explanations for the absence of the predicted effects and some of these will be considered here with respect to each procedure in turn.

The literature on WM training is mixed and a number of recent reviews have highlighted limitations in the reliability of the distant transfer of training in particular (Harrison et al., 2013; Melby-Lervg Hulme, 2015; Shipstead, Redick, Engle, 2012; Simons et al., 2016). A lack of standardised and reliable training protocols, including adequate controls and validated schedules, may be responsible for the inconsistent results in the literature (Morrison Chein, 2011) and, in light of this, the present study used an established dual n-back training program (Max Owens et al., 2013). Despite the mixed literature, it is difficult to ignore previous findings that suggest generalized improvements to fluid intelligence following training and more widely replicated near 
a)

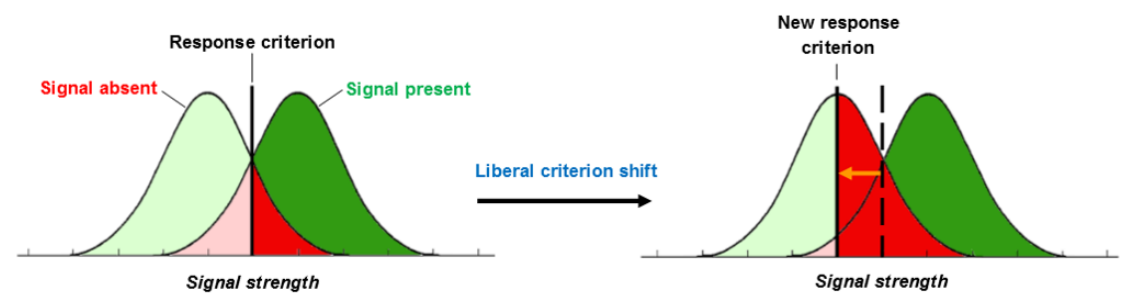

b)
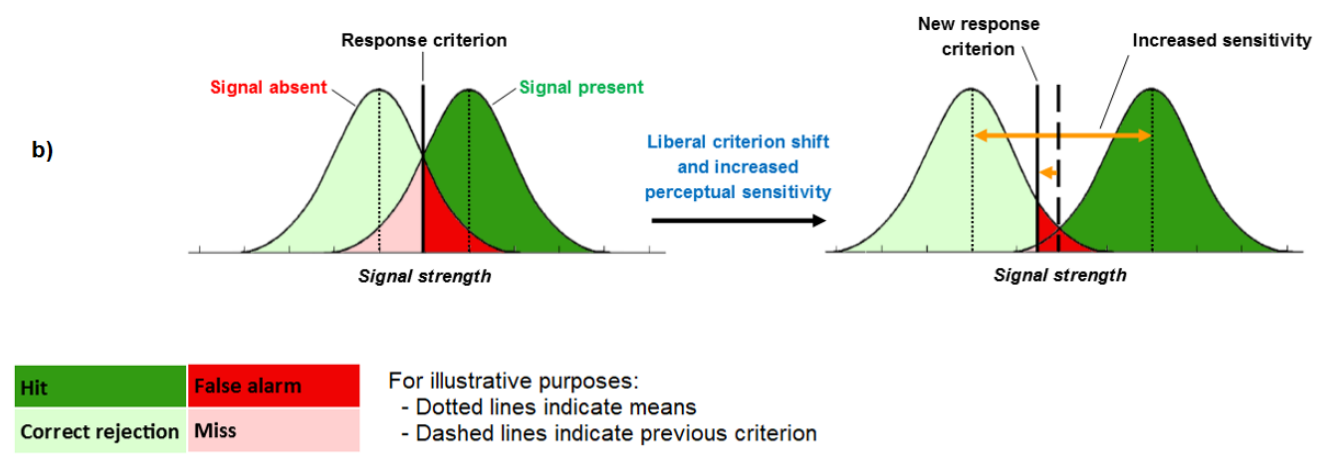

Figure 13. Hypothetical signal and noise distributions illustrating: (a) the effect of a shift in response criterion; and (b) the combined effect of a criterion shift and increased sensitivity.

transfer effects (Au et al., 2014, 2015; Greenwood Parasuraman, 2016; Jaeggi et al., 2008). If the present results had indicated a lack of transfer to the visual search task in the presence of a range of near transfer effects on the other WM and attention tasks, then one might reasonably explore explanations for the lack of distant transfer that assumed a basic, if limited, level of training effectiveness, for example, underlying individual differences in neuroanatomy (Simon, Skinner, Ziegler, 2016). It is possible that a greater sample size may have revealed such effects, however, this still seems unlikely given the absence of near transfer to other basic WM tasks, in particular the digit span, and previous reliable findings of such near transfer with similar sample sizes. The WM training arm of the present study involved 20 participants. This is actually marginally greater than the mean number of participants (19.96) across the 20 studies reviewed by Au et al. $(2014,2015)$ in their meta-analyses which found significant transfer effects. This suggests that, in itself, sample size cannot fully explain the present null results. Participants in the active training group did not benefit to the extent that was anticipated and this may reflect the shortcomings of an approach that allows greater flexibility in when and where participants completed their training. While training performance and time-of-day was monitored, this is not necessarily sufficient to ensure high levels of engagement and motivation and it is reasonable to expect that lab-based training, while sacrificing flexibility, may have produced better outcomes.

The data from the tDCS arm of the study also provided stronger support for practice and null effects than for stimulation effects. The fact that tDCS was found to have no effect upon the search task or the WM and attention task suggests a more fundamental explanation. One such explanation, in the context of the present experimental method, is the timing and duration of the tasks following stimulation. The visual search task had the longest duration and was completed immediately following stimulation, the other 
WM and attention tasks were completed after this. Changes in cortical excitability have been shown to persist for 1.5 hours following stimulation at $1 \mathrm{~mA}$ for 13 minutes (Nitsche Paulus, 2001), a weaker current and a shorter duration than the stimulation delivered in the present study. However, the effects of stimulation do diminish over time and one would reasonably expect a reduced likelihood of observing any significant effects of stimulation in tasks performed towards the end of a testing session compared to the beginning.

Early tDCS research established clear directional predictions about the excitatory and inhibitory effects of anodal and cathodal stimulation respectively (Nitsche Paulus, 2000). However, as tDCS has been applied to a greater range of brain regions and with different electrode configurations, it has become clear that its effects cannot be so clearly distinguished and that results may influenced by the present state of underlying networks, by inter-individual differences and, in unilateral montages, by the interhemispheric balance of activity (Fecteau et al., 2007; Tremblay et al., 2014). Similar to other recent studies, the present study employed a bilateral montage, which should minimize variation in the balance of activity between hemispheres (Miler et al., 2017; Nelson, McKinley, Golob, Warm, Parasuraman, 2014; Oliveira et al., 2013) and like 14 out of 16 studies in a recent meta-analysis, the present study focused exclusively on anodal stimulation of the left DLPFC (Hill, Fitzgerald, Hoy, 2016). However, two recent reviews by another group of authors, one examining 117 studies and the other 198 studies, have even suggested that single-session tDCS has no reliable effect upon cognitive performance (Horvath, Forte, Carter, 2015b, 2015a), although this may be attributable to issues with inter-subject variability (Horvath, Carter, Forte, 2014) and the resting brain state dependency of stimulation effects (Silvanto, Muggleton, Walsh, 2008).

\section{Conclusions and Implications}

In summary, the present study supports and extends previous findings obtained from the same search and monitoring task. Relative to previous work, higher target prevalence was found to improve performance and facilitate the speeded predictive detection of targets, which represents a novel type of prevalence effect. We suggest that a higher level of target prevalence increased the validity of target predictive distractors as cues to forthcoming target onsets. This has implications for the visualization of data in monitoring tasks. Dependent upon the level of target prevalence in a given task, displays that support a predictive monitoring strategy could allow for faster responses and, in tasks with lower target prevalence, displays could likely be simplified with no cost to RTs.

Increased target prevalence also affected the relationship between individual differences in IU (including its interaction with verbal WMC) and target detection, with an effect being observed in terms of hit rate rather than false alarm rate. We provide an account of these findings in terms of a criterion shift, driven by IU, and a change in perceptual sensitivity, driven by verbal WMC. Critically, we found evidence against WM training and tDCS being effective in improving performance in the search task or other measures of WMC and attentional control. These results are informative and provide useful methodological considerations for the application of WM training and tDCS in future experimental work, highlighting obstacles that would need to be overcome if these methods were ever to be integrated into training for real-world monitoring tasks.

In addition to the proposed theoretical account of the effects of IU and verbal WMC, 
the pattern of results around these specific individual factors has practical implications for real-world visual monitoring tasks. For example, when selecting personnel for search and monitoring tasks, IU and verbal WMC represent characteristics that could be used to identify individuals who are likely to perform well at any given level of target prevalence. Both IU and verbal WMC can be assessed very quickly and easily, IU with a 12-item questionnaire and verbal WMC with a digit span, or, as in Muhl-Richardson et al. (2018), an n-back type task. Maximizing performance according to individual aptitude could be particularly important in safety critical monitoring tasks, such as those carried out by military radar operators, where an inaccurate or slow response could carry a significant cost. 


\section{References}

Anderson, E. J., Mannan, S. K., Rees, G., Sumner, P., and Kennard, C. (2008). A role for spatial and nonspatial working memory processes in visual search. Experimental Psychology, $55(5): 301-312$.

Au, J., Buschkuehl, M., Duncan, G. J., and Jaeggi, S. M. (2015). There is no convincing evidence that working memory training is NOT effective: A reply to melby-lervåg and hulme (2015). Psychonomic Bulletin \& Review, 23(1):331-337.

Au, J., Sheehan, E., Tsai, N., Duncan, G. J., Buschkuehl, M., and Jaeggi, S. M. (2014). Improving fluid intelligence with training on working memory: a meta-analysis. Psychonomic Bulletin \& Review, 22(2):366-377.

Bates, D., Mchler, M., Bolker, B., and Walker, S. (2015). Fitting linear mixed-effects models usinglme4. Journal of Statistical Software, 67(1).

Berryhill, M. E. and Jones, K. T. (2012). tDCS selectively improves working memory in older adults with more education. Neuroscience Letters, 521(2):148-151.

Birrell, J., Meares, K., Wilkinson, A., and Freeston, M. (2011). Toward a definition of intolerance of uncertainty: A review of factor analytical studies of the intolerance of uncertainty scale. Clinical Psychology Review, 31(7):1198-1208.

Boggio, P. S., Bermpohl, F., Vergara, A. O., Muniz, A. L., Nahas, F. H., Leme, P. B., Rigonatti, S. P., and Fregni, F. (2007). Go-no-go task performance improvement after anodal transcranial DC stimulation of the left dorsolateral prefrontal cortex in major depression. Journal of Affective Disorders, 101(1-3):91-98.

Boggio, P. S., Ferrucci, R., Rigonatti, S. P., Covre, P., Nitsche, M., Pascual-Leone, A., and Fregni, F. (2006). Effects of transcranial direct current stimulation on working memory in patients with parkinsons disease. Journal of the Neurological Sciences, 249(1):31-38.

Booth, R. W., Mackintosh, B., and Sharma, D. (2017). Working memory regulates trait anxiety-related threat processing biases. Emotion, 17(4):616-627.

Brehmer, Y., Westerberg, H., and Bckman, L. (2012). Working-memory training in younger and older adults: training gains, transfer, and maintenance. Frontiers in Human Neuroscience, 6.

Broadway, J. M. and Engle, R. W. (2011). Individual differences in working memory capacity and temporal discrimination. PLoS ONE, 6(10):e25422.

Brunoni, A. R. and Vanderhasselt, M.-A. (2014). Working memory improvement with noninvasive brain stimulation of the dorsolateral prefrontal cortex: A systematic review and meta-analysis. Brain and Cognition, 86:1-9.

Carleton, R. N., Norton, M. P. J., and Asmundson, G. J. (2007). Fearing the unknown: A short version of the intolerance of uncertainty scale. Journal of Anxiety Disorders, 21(1):105117.

Clarke, P. J., Browning, M., Hammond, G., Notebaert, L., and MacLeod, C. (2014). The causal role of the dorsolateral prefrontal cortex in the modification of attentional bias: Evidence from transcranial direct current stimulation. Biological Psychiatry, 76(12):946-952.

Dedoncker, J., Brunoni, A. R., Baeken, C., and Vanderhasselt, M.-A. (2016). The effect of the interval-between-sessions on prefrontal transcranial direct current stimulation (tDCS) on 
cognitive outcomes: a systematic review and meta-analysis. Journal of Neural Transmission, 123(10):1159-1172.

Derakshan, N. and Eysenck, M. W. (2009). Anxiety, processing efficiency, and cognitive performance. European Psychologist, 14(2):168-176.

Endres, M. J., Rickert, M. E., Bogg, T., Lucas, J., and Finn, P. R. (2011). Externalizing psychopathology and behavioral disinhibition: Working memory mediates signal discriminability and reinforcement moderates response bias in approach-avoidance learning. Journal of $A b$ normal Psychology, 120(2):336-351.

Eysenck, M. W. and Derakshan, N. (2011). New perspectives in attentional control theory. Personality and Individual Differences, 50(7):955-960.

Eysenck, M. W., Derakshan, N., Santos, R., and Calvo, M. G. (2007). Anxiety and cognitive performance: Attentional control theory. Emotion, 7(2):336-353.

FAN, J., MCCANDLISS, B., FOSSEllA, J., FLOMBAUM, J., and POSNER, M. (2005). The activation of attentional networks. NeuroImage, 26(2):471-479.

Fan, J., McCandliss, B. D., Sommer, T., Raz, A., and Posner, M. I. (2002). Testing the efficiency and independence of attentional networks. Journal of Cognitive Neuroscience, $14(3): 340-347$.

Fecteau, S., Knoch, D., Fregni, F., Sultani, N., Boggio, P., and Pascual-Leone, A. (2007). Diminishing risk-taking behavior by modulating activity in the prefrontal cortex: A direct current stimulation study. Journal of Neuroscience, 27(46):12500-12505.

Fergus, T. A., Bardeen, J. R., and Wu, K. D. (2012). Intolerance of uncertainty and uncertainty-related attentional biases: Evidence of facilitated engagement or disengagement difficulty? Cognitive Therapy and Research, 37(4):735-741.

Fergus, T. A. and Carleton, R. N. (2016). Intolerance of uncertainty and attentional networks: Unique associations with alerting. Journal of Anxiety Disorders, 41:59-64.

Fregni, F., Boggio, P. S., Nitsche, M., Bermpohl, F., Antal, A., Feredoes, E., Marcolin, M. A., Rigonatti, S. P., Silva, M. T., Paulus, W., and Pascual-Leone, A. (2005). Anodal transcranial direct current stimulation of prefrontal cortex enhances working memory. Experimental Brain Research, 166(1):23-30.

Godwin, H. J., Menneer, T., Riggs, C. A., Cave, K. R., and Donnelly, N. (2014). Perceptual failures in the selection and identification of low-prevalence targets in relative prevalence visual search. Attention, Perception, 6 Psychophysics, 77(1):150-159.

Greenwood, P. M. and Parasuraman, R. (2016). The mechanisms of far transfer from cognitive training: Review and hypothesis. Neuropsychology, 30(6):742-755.

Hadwin, J., Visu-Petra, L., Muris, P., Derakshan, N., and Macleod, C. (2016). Introduction to the special issue: Understanding the role of attentional control in the development of anxiety in childhood, adolescence and across the lifespan. Journal of Experimental Psychopathology, $7(3): 204380871600700$.

Harrison, T. L., Shipstead, Z., Hicks, K. L., Hambrick, D. Z., Redick, T. S., and Engle, R. W. (2013). Working memory training may increase working memory capacity but not fluid intelligence. Psychological Science, 24(12):2409-2419.

Hepsomali, P., Hadwin, J. A., Liversedge, S. P., and Garner, M. (2017). Pupillometric and saccadic measures of affective and executive processing in anxiety. Biological Psychology, $127: 173-179$. 
Hill, A. T., Fitzgerald, P. B., and Hoy, K. E. (2016). Effects of anodal transcranial direct current stimulation on working memory: A systematic review and meta-analysis of findings from healthy and neuropsychiatric populations. Brain Stimulation, 9(2):197-208.

Horvath, J. C., Carter, O., and Forte, J. D. (2014). Transcranial direct current stimulation: five important issues we arent discussing (but probably should be). Frontiers in Systems Neuroscience, 8.

Horvath, J. C., Forte, J. D., and Carter, O. (2015). Evidence that transcranial direct current stimulation (tDCS) generates little-to-no reliable neurophysiologic effect beyond MEP amplitude modulation in healthy human subjects: A systematic review. Neuropsychologia, $66: 213-236$.

Iyer, M. B., Mattu, U., Grafman, J., Lomarev, M., Sato, S., and Wassermann, E. M. (2005). Safety and cognitive effect of frontal DC brain polarization in healthy individuals. Neurology, 64(5):872-875.

Jaeggi, S. M., Buschkuehl, M., Jonides, J., and Perrig, W. J. (2008). Improving fluid intelligence with training on working memory. Proceedings of the National Academy of Sciences, 105(19):6829-6833.

Jaeggi, S. M., Buschkuehl, M., Jonides, J., and Shah, P. (2012). Cogmed and working memory training - current challenges and the search for underlying mechanisms. Journal of Applied Research in Memory and Cognition, 1(3):211-213.

JASP Team. Jasp (version 0.9).

Keeser, D., Padberg, F., Reisinger, E., Pogarell, O., Kirsch, V., Palm, U., Karch, S., Mller, H.-J., Nitsche, M., and Mulert, C. (2011). Prefrontal direct current stimulation modulates resting EEG and event-related potentials in healthy subjects: A standardized low resolution tomography (sLORETA) study. NeuroImage, 55(2):644-657.

Kincses, T. Z., Antal, A., Nitsche, M. A., Bártfai, O., and Paulus, W. (2004). Facilitation of probabilistic classification learning by transcranial direct current stimulation of the prefrontal cortex in the human. Neuropsychologia, 42(1):113-117.

Klingberg, T., Forssberg, H., and Westerberg, H. (2002). Training of working memory in children with ADHD. Journal of Clinical and Experimental Neuropsychology, 24(6):781-791.

Kunar, M. A. and Watson, D. G. (2011). Visual search in a multi-element asynchronous dynamic (MAD) world. Journal of Experimental Psychology: Human Perception and Performance, 37(4):1017-1031.

Kunar, M. A. and Watson, D. G. (2014). When are abrupt onsets found efficiently in complex visual search? evidence from multielement asynchronous dynamic search. Journal of Experimental Psychology: Human Perception and Performance, 40(1):232-252.

Lara, A. H. and Wallis, J. D. (2015). The role of prefrontal cortex in working memory: A mini review. Frontiers in Systems Neuroscience, 9.

Melby-Lervåg, M. and Hulme, C. (2015). There is no convincing evidence that working memory training is effective: A reply to au et al. (2014) and karbach and verhaeghen (2014). Psychonomic Bulletin \& Review, 23(1):324-330.

Miler, J. A., Meron, D., Baldwin, D. S., and Garner, M. (2017). The effect of prefrontal transcranial direct current stimulation on attention network function in healthy volunteers. Neuromodulation: Technology at the Neural Interface, 21(4):355-361. 
Morrison, A. B. and Chein, J. M. (2010). Does working memory training work? the promise and challenges of enhancing cognition by training working memory. Psychonomic Bulletin $\mathcal{E}$ Review, 18(1):46-60.

Muhl-Richardson, A., Godwin, H. J., Garner, M., Hadwin, J. A., Liversedge, S. P., and Donnelly, N. (2018). Individual differences in search and monitoring for color targets in dynamic visual displays. Journal of Experimental Psychology: Applied, 24(4):564-577.

Mulquiney, P. G., Hoy, K. E., Daskalakis, Z. J., and Fitzgerald, P. B. (2011). Improving working memory: Exploring the effect of transcranial random noise stimulation and transcranial direct current stimulation on the dorsolateral prefrontal cortex. Clinical Neurophysiology, 122(12):2384-2389.

Nelson, J. T., McKinley, R. A., Golob, E. J., Warm, J. S., and Parasuraman, R. (2014). Enhancing vigilance in operators with prefrontal cortex transcranial direct current stimulation (tDCS). NeuroImage, 85:909-917.

Nitsche, M. A., Cohen, L. G., Wassermann, E. M., Priori, A., Lang, N., Antal, A., Paulus, W., Hummel, F., Boggio, P. S., Fregni, F., and Pascual-Leone, A. (2008). Transcranial direct current stimulation: State of the art 2008. Brain Stimulation, 1(3):206-223.

Nitsche, M. A. and Paulus, W. (2000). Excitability changes induced in the human motor cortex by weak transcranial direct current stimulation. The Journal of Physiology, 527(3):633639.

Nitsche, M. A. and Paulus, W. (2001). Sustained excitability elevations induced by transcranial DC motor cortex stimulation in humans. Neurology, 57(10):1899-1901.

Ohn, S. H., Park, C.-I., Yoo, W.-K., Ko, M.-H., Choi, K. P., Kim, G.-M., Lee, Y. T., and Kim, Y.-H. (2008). Time-dependent effect of transcranial direct current stimulation on the enhancement of working memory. NeuroReport, 19(1):43-47.

Oliveira, J. F., Zanão, T. A., Valiengo, L., Lotufo, P. A., Benseñor, I. M., Fregni, F., and Brunoni, A. R. (2013). Acute working memory improvement after tDCS in antidepressant-free patients with major depressive disorder. Neuroscience Letters, 537:60-64.

Owens, M., Koster, E. H. W., and Derakshan, N. (2011). Impaired filtering of irrelevant information in dysphoria: an ERP study. Social Cognitive and Affective Neuroscience, 7(7):752763.

Owens, M., Koster, E. H. W., and Derakshan, N. (2013). Improving attention control in dysphoria through cognitive training: Transfer effects on working memory capacity and filtering efficiency. Psychophysiology, 50(3):297-307.

Owens, M., Stevenson, J., Hadwin, J. A., and Norgate, R. (2012). When does anxiety help or hinder cognitive test performance? the role of working memory capacity. British Journal of Psychology, 105(1):92-101.

Pergolizzi, D. and Chua, E. F. (2017). Increased contextual cue utilization with tDCS over the prefrontal cortex during a recognition task. Brain Research, 1655:1-9.

R, S., G, B., and SM, C. (2016). Bilateral transcranial direct-current stimulation (tDCS) of dorsolateral prefrontal cortex during specific working memory tasks. International Journal of Physical Medicine $\&$ Rehabilitation, 4(5).

Richards, H. J., Hadwin, J. A., Benson, V., Wenger, M. J., and Donnelly, N. (2011). The influence of anxiety on processing capacity for threat detection. Psychonomic Bulletin 6 Review, 18(5):883-889. 
Rosenkranz, K., Nitsche, M. A., Tergau, F., and Paulus, W. (2000). Diminution of traininginduced transient motor cortex plasticity by weak transcranial direct current stimulation in the human. Neuroscience Letters, 296(1):61-63.

Rossi, A. F., Pessoa, L., Desimone, R., and Ungerleider, L. G. (2008). The prefrontal cortex and the executive control of attention. Experimental Brain Research, 192(3):489-497.

Rouder, J. N., Haaf, J. M., and Aust, F. (2017). From theories to models to predictions: A bayesian model comparison approach. Communication Monographs, 85(1):41-56.

Sari, B. A., Koster, E. H., Pourtois, G., and Derakshan, N. (2016). Training working memory to improve attentional control in anxiety: A proof-of-principle study using behavioral and electrophysiological measures. Biological Psychology, 121:203-212.

Scarince, C. and Hout, M. C. (2018). Cutting through the MADness: Expectations about what a target is doing impact how likely it is to be found in dynamic visual displays. Quarterly Journal of Experimental Psychology, 71(11):2342-2354.

Schwark, J., Sandry, J., and Dolgov, I. (2013). Evidence for a positive relationship between working-memory capacity and detection of low-prevalence targets in visual search. Perception, $42(1): 112-114$.

Sheehan, D., Lecrubier, Y., Sheehan, K. H., Janavs, J., Weiller, E., Keskiner, A., Schinka, J., Knapp, E., Sheehan, M., and Dunbar, G. (1997). The validity of the mini international neuropsychiatric interview (MINI) according to the SCID-p and its reliability. European Psychiatry, 12(5):232-241.

Shipstead, Z., Redick, T. S., and Engle, R. W. (2012). Is working memory training effective? Psychological Bulletin, 138(4):628-654.

Silvanto, J., Muggleton, N., and Walsh, V. (2008). State-dependency in brain stimulation studies of perception and cognition. Trends in Cognitive Sciences, 12(12):447-454.

Simon, A. J., Skinner, S. N., and Ziegler, D. A. (2016). Training working memory: Anatomy matters. The Journal of Neuroscience, 36(30):7805-7806.

Simons, D. J., Boot, W. R., Charness, N., Gathercole, S. E., Chabris, C. F., Hambrick, D. Z., and Stine-Morrow, E. A. L. (2016). Do "brain-training" programs work? Psychological Science in the Public Interest, 17(3):103-186.

Smirni, D., Turriziani, P., Mangano, G. R., Cipolotti, L., and Oliveri, M. (2015). Modulating memory performance in healthy subjects with transcranial direct current stimulation over the right dorsolateral prefrontal cortex. PLOS ONE, 10(12):e0144838.

Soto, D., Wriglesworth, A., Bahrami-Balani, A., and Humphreys, G. W. (2010). Working memory enhances visual perception: Evidence from signal detection analysis. Journal of Experimental Psychology: Learning, Memory, and Cognition, 36(2):441-456.

Teo, F., Hoy, K. E., Daskalakis, Z. J., and Fitzgerald, P. B. (2011). Investigating the role of current strength in tDCS modulation of working memory performance in healthy controls. Frontiers in Psychiatry, 2.

Tremblay, S., Lepage, J.-F., Latulipe-Loiselle, A., Fregni, F., Pascual-Leone, A., and Théoret, H. (2014). The uncertain outcome of prefrontal tDCS. Brain Stimulation, 7(6):773-783.

Unsworth, N. and Engle, R. W. (2007). The nature of individual differences in working memory capacity: Active maintenance in primary memory and controlled search from secondary memory. Psychological Review, 114(1):104-132. 
Wagenmakers, E.-J., Marsman, M., Jamil, T., Ly, A., Verhagen, J., Love, J., Selker, R., Gronau, Q. F., Šmíra, M., Epskamp, S., Matzke, D., Rouder, J. N., and Morey, R. D. (2017). Bayesian inference for psychology. part i: Theoretical advantages and practical ramifications. Psychonomic Bulletin \& Review, 25(1):35-57.

Westerberg, H. and Klingberg, T. (2007). Changes in cortical activity after training of working memory — a single-subject analysis. Physiology \& B Behavior, 92(1-2):186-192.

Wolfe, J. M., Horowitz, T. S., and Kenner, N. M. (2005). Rare items often missed in visual searches. Nature, 435(7041):439-440.

Wolfe, J. M., Horowitz, T. S., Wert, M. J. V., Kenner, N. M., Place, S. S., and Kibbi, N. (2007). Low target prevalence is a stubborn source of errors in visual search tasks. Journal of Experimental Psychology: General, 136(4):623-638.

Wolfe, J. M. and Wert, M. J. V. (2010). Varying target prevalence reveals two dissociable decision criteria in visual search. Current Biology, 20(2):121-124.

Woodman, G. F. and Luck, S. J. (2004). Visual search is slowed when visuospatial working memory is occupied. Psychonomic Bulletin \& Review, 11(2):269-274.

Woods, D. L., Kishiyama, M. M., Yund, E. W., Herron, T. J., Edwards, B., Poliva, O., Hink, R. F., and Reed, B. (2010). Improving digit span assessment of short-term verbal memory. Journal of Clinical and Experimental Neuropsychology, 33(1):101-111.

Zwissler, B., Sperber, C., Aigeldinger, S., Schindler, S., Kissler, J., and Plewnia, C. (2014). Shaping memory accuracy by left prefrontal transcranial direct current stimulation. Journal of Neuroscience, 34(11):4022-4026. 